\title{
The Oxford Magnetic Suspension and Balance System: A Brief History and Development Status
}

\author{
Luke J. Doherty,* Nathan Donaldson, ${ }^{\dagger}$ Andrew K. Owen, \\ Oxford Thermofluids Institute, University of Oxford, Oxford, United Kingdom, OX1 3PJ
}

This paper traces the history of the Oxford Magnetic Suspension and Balance System, from its initial development in the 1960s through to current times. Developed in conjunction with the Oxford Low Density Tunnel, the balance has been a key instrument throughout its history for investigating aerodynamic forces at high Mach numbers, low density flows across the continuum, slip and transition regimes. An initial balance was developed as a 2-axis system with control over only lift and drag. Following its success, a second balance was designed to control lift, drag and additionally pitch. This enabled more complex geometries, such as a re-entry Aerobrake model and NASA's X-43 hypersonic demonstrator to be investigated. The evolution of the electro-mechanical design of each balance and the associated model attitude detection systems are described in this paper. Operational issues encountered with the system and sample results from past studies are also presented and discussed. The paper concludes with an outlook to the future development and application of the magnetic suspension balance system within the Oxford Low Density Tunnel.

\section{Nomenclature}
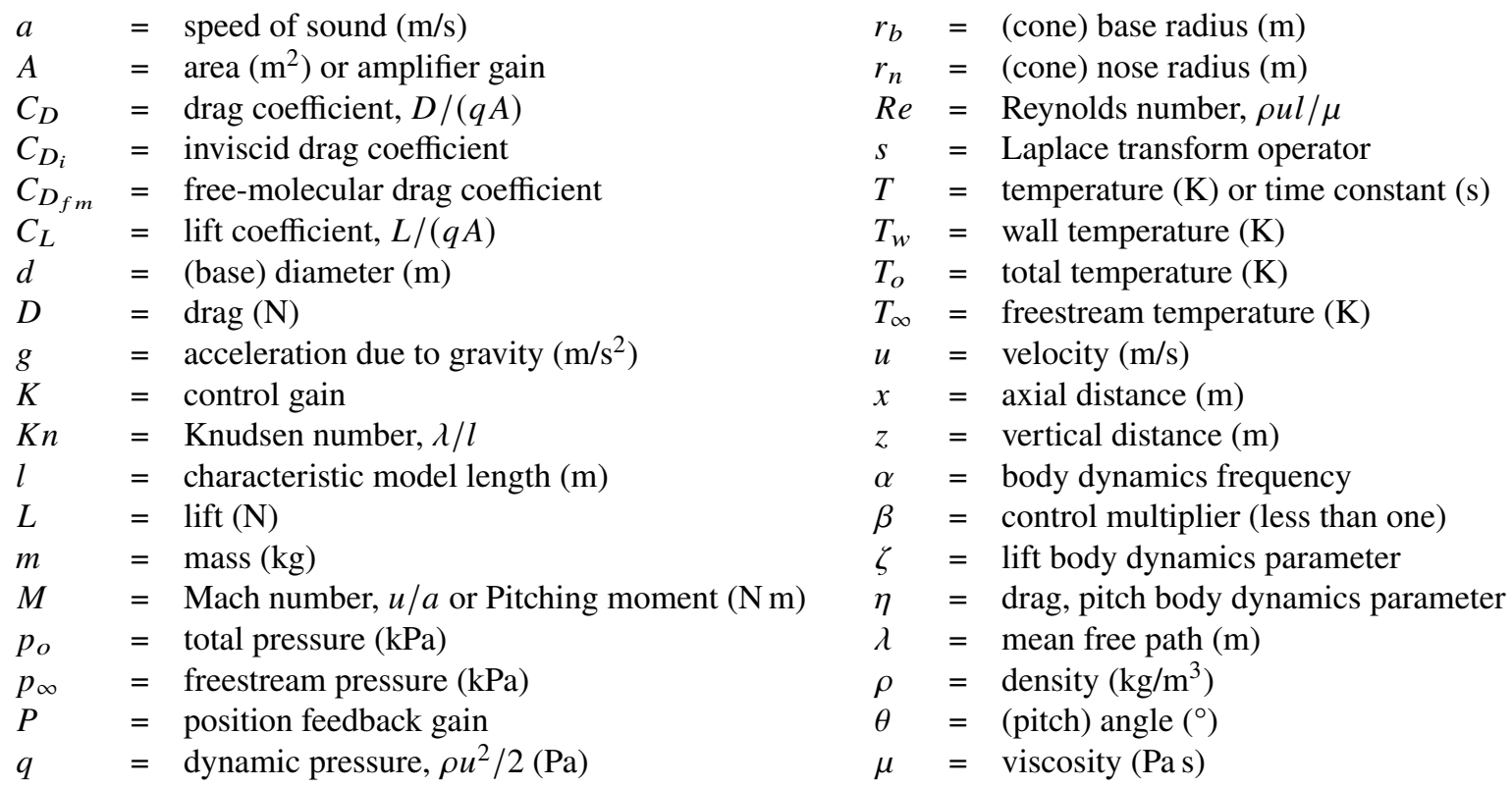

\section{Introduction}

In the words of Haslam-Jones [1] magnetic suspension is the most "beautiful" technologically feasible method of suspending wind tunnel models. First demonstrated by researchers from the French aerospace laboratory ONERA in 1957 [2], magnetic suspension and balance systems (MSBSs) were soon being applied to wind tunnels by groups around

\footnotetext{
* Senior Research Associate, Department of Engineering Sciences, AIAA Member, luke.doherty@eng.ox . ac .uk

${ }^{\dagger}$ Post-doctoral Research Assistant, Department of Engineering Sciences

${ }_{\ddagger}^{\ddagger}$ Post-doctoral Research Assistant, Department of Engineering Sciences
} 
the world [3, Section 1]. Growth in the area was rapid with researchers pursuing the possibility of making accurate aerodynamic measurements on models, free from any adverse mounting or sting-interaction effects. By end of 1965, which marks the start of Altmann's [4] effort to develop an MSBS at Oxford, 44 research papers had been published on the application of MSBSs to wind tunnels and on advancing the technology [5]. However, as Altmann noted, the technique is only as useful as the measurements it enables. In the UK at that time the University of Southampton via the research of Goodyer [6] were developing a 6-component balance for the measurement of dynamic stability derivatives. Goodyer would remain at Southampton, staying at the forfront of MSBS technology development throughout his career. Concurrently, in the 1960s Crane [7] was using a 5-axis balance developed at the Royal Aircraft Establishment (RAE) for use with a $7 \mathrm{in.} \times 7 \mathrm{in}$. hypersonic wind tunnel, to study the near wake region behind conical models. It was during the 1960s and early 1970s, at the height of the space-race, that experimental measurements of the aerodynamic forces on simple geometries (such as spheres and cones) travelling at high-speeds and high-altitudes were of significant interest. Computers and the numerical simulation of hypersonic flows were in their infancy and for rarefied transitional flows between the continuum and free-molecular limits, analytical solutions were intractable because of complex merged viscous-shock-layers and non-equilibrium thermochemical effects. It was not until the invention and acceptance of Direct Simulation Monte Carlo (DSMC) methods [8] that numerical simulation of transitional flow fields became possible. Thus during this period, aerodynamic data in the hypersonic rarefied transition regime existed solely in the domain of the experimentalist [1, pg. 88].

In 1963 the University of Oxford's Department of Engineering Science had taken delivery of the Low Density Tunnel (LDT), a continuous, hypersonic, rarefied flow facility. In rarefied flows the low gas density, large viscous interaction regions and very small models sizes required by scaling laws, make measurement of aerodynamic forces using traditional sting-based methods very difficult. In the second half of the 1960s the LDT was used to understand wake flow behind cylindrical models [9], however researchers saw the utility of MSBS technology for this facility and flow regime. Application of MSBS technology to LDT would come to define the research output from the facility over the next 30 years.

This paper describes the development and usage of MSBSs at the University of Oxford, from the initial development of a 2-axis system through to the current status of our 3-axis system. Section II begins by providing a brief overview of the Oxford LDT, it being the wind tunnel facility that the MSBSs were designed for. Section III continues the paper with a description of the work of Altmann [4, 10] who developed Oxford's first MSBS and a description of Hadjimichalis' [11] experiments, who used the balance to characterize drag on a sphere from continuum to free-molecular flow. Section IV discusses the three-component MSBS developed by Haslam-Jones [1] and the experimental campaigns of Dahlen [12] and Smith and Lord [13] who investigated cone drag in rarefied flows. The work of Owen [14], who made several key improvements to the three-component balance is presented in Section V. The current status and future plans for the balance are described in Section VI before the paper concludes in Section VII.

\section{The Low Density Tunnel: Overview}

A discussion of the Oxford MSBS cannot be complete without knowledge of the Low Density Tunnel, as it was this wind tunnel facility that the MSBS was specifically developed for and which imposed several design constraints. A photograph and schematic of the Oxford LDT are provided in Figure 1. The facility operates as a free-jet, open-circuit, continuous-flow facility that is capable of generating hypersonic flows primarily in the slip and transition regimes. Delivered to the Department of Engineering Science in 1963, the facility is comprised of three major sub-systems: (1) the gas delivery system, (2) the test section, and (3) the pumping system. The test gas, which may be any inert gas,* is admitted to a stagnation chamber via a mass flow controller and in-line heater. The stagnation chamber itself is also heated to reduce thermal losses. Acceleration of the gas from the stagnation chamber into the test section is currently achieved using a contoured Mach 6 nozzle, although conical nozzles and orifices plates have also been used in the past $[1,11]$. The Mach 6 nozzle currently installed has a throat diameter of $9.65 \mathrm{~mm}$, an exit diameter of $107.5 \mathrm{~mm}$ and produces a core flow region of $<40 \mathrm{~mm}$ diameter, depending on the test condition.

The test section consists of a large cylindrical vessel approximately $1540 \mathrm{~mm}$ in diameter and $1500 \mathrm{~mm}$ length. The axis of the cylinder is perpendicular to that of the nozzle and the test section may be accessed from either side by full-size dished doors. Downstream, the test section joins to a vertical settling chamber that is isolated from the pumping system by a high vacuum valve. The pumping system was one of approximately four identical systems produced by Edwards Vacuum at the time of manufacture. It consists of an Edwards 100B4 ejector vapour booster pump backed by an 1R80 Roots-style mechanical booster pump and three HISC3000 rotary piston pumps. As a whole, the system is

\footnotetext{
*Combustible gases can also be used with careful dilution upstream of the backing pumps.
} 


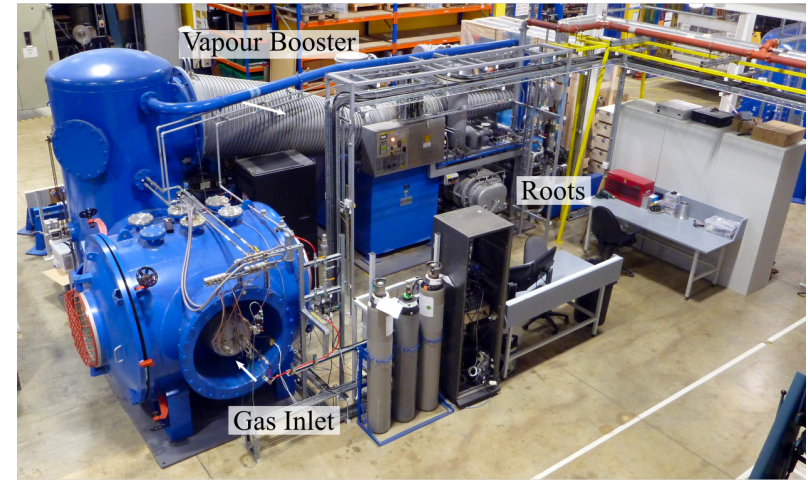

(a) Photograph of the LDT as currently installed at the Oxford Thermofluids Institute

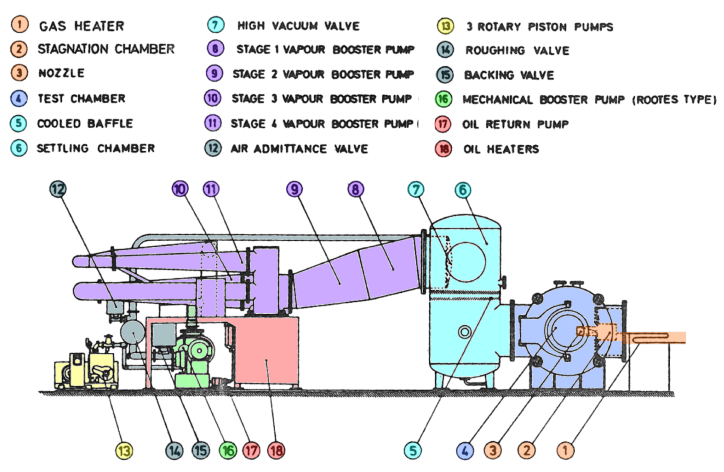

(b) Facility schematic

Fig. 1. The Oxford Low Density Tunnel

capable of pumping $22000 \mathrm{l} / \mathrm{s}$ at back pressures up to $\approx 2 \mathrm{~Pa}$. With no gas admittance, the system is capable of achieving an ultimate pressure of $<0.01 \mathrm{~Pa}$. The facility has recently been refurbished and recommissioned, further details of which may be found in Donaldson et al. [15] and Donaldson [16].

Although the vapour booster has a large pumping speed, the mass flow rate is limited to approximately $0.5 \mathrm{~g} / \mathrm{s}$ of air (at $200 \mathrm{~Pa}^{\dagger}$ ). This places limits on both the maximum stagnation pressure and the nozzle throat or orifice size used for testing. A small nozzle requires similarly small models. For the current Mach 6 nozzle, models are limited to $<20 \mathrm{~mm}$ based on the projected frontal area. In rarefied aerodynamics, the Knudsen number, defined as

$$
K n=\frac{\lambda}{l}
$$

where $\lambda$ is the gas molecule mean free path and $l$ is a characteristic model length, is an important dimensionless number which indicates the relative dominance of molecule-molecule interactions vs molecule-surface interactions in the flow field. Knudsen numbers less than 0.001 represent continuum flow for which the Navier-Stokes equations hold and molecule-molecule interactions dominate. At the opposite end of the scale, Knudsen numbers larger than 10 represent free-molecular flow in which the collisionless Boltzmann equation holds and the flow is dominated by molecule-surface interactions. Between these limits lie the slip regime, taken to be from $K n=0.001$ to 0.1 and the transition regime which covers the range $K n=0.1$ to 10. From the definition of Knudsen number it is obvious that a range of Knudsen numbers can be achieved experimentally simply by altering the model length scale while keeping the test condition fixed. Small models and rarefied flow conditions result in aerodynamic loads on the order of a few milli-Newton. Forces of this magnitude are difficult to measure accurately with sting mounted models and strain-gauge based force balances. Experiments are subject to further uncertainty due to strong viscous effects and large regions of influence that occur under rarefied flow conditions. Magnetic suspension and balance systems thus offer unique advantages for hypersonic rarefied aerodynamic testing.

Altmann [4] and Haslam-Jones [1] each identified a number of constraints placed on the design of a MSBS by the LDT layout and operation. These included -

1) restrictions on the overall size of the MSBS,

2) that the magnets be able operate under medium/high vacuum conditions, ideally for long periods (several hours),

3) that the MSBS be designed for a horizontal air flow,

4) that experimental models are able to be accurately positioned and deposited in the magnetic field without opening the test section,

5) that the spacing of the magnets sufficiently accommodate the $110 \mathrm{~mm}$ exit diameter of the Mach 6 nozzle and not interfere with the flow, and

6) that the required voltages be achievable with power transistors and technology available at the time.

However, despite these contraints, two MSBSs were successfully designed and commissioned over the course of the 1970s and 1980s.

\footnotetext{
${ }^{\dagger}$ There is a slight increase in the pumping speed to $\approx 25000 \mathrm{l} / \mathrm{s}$ at $\approx 400 \mathrm{~Pa}$ inlet pressure, corresponding to a maximum mass flow of $1.2 \mathrm{~g} / \mathrm{s}$ of air.
} 


\section{Initial Development, the early 1970s}

\section{A. Balance Description}

The development of Oxford's first magnetic suspension system was undertaken by Altmann $[4,10]$ during and following his doctoral studies. At that time, the only other operational MSBS in the UK were those at the University of Southampton [6] and the Royal Aircraft Establishment [7]. At Oxford, the need for an MSBS arose from the desire to measure the drag coefficient of a sphere in the free-molecular flow regime [4]. Achieving the desired range of Knudsen number entailed using spheres as small as $1.58 \mathrm{~mm}$ (1/16 in.) [11] which practically excluded the use of sting-based measurement methods.

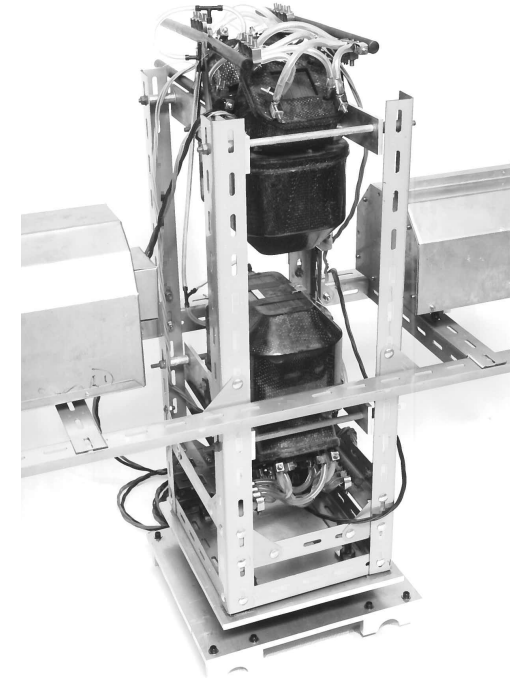

(a) Photograph of the completed balance assembly, circa 1973

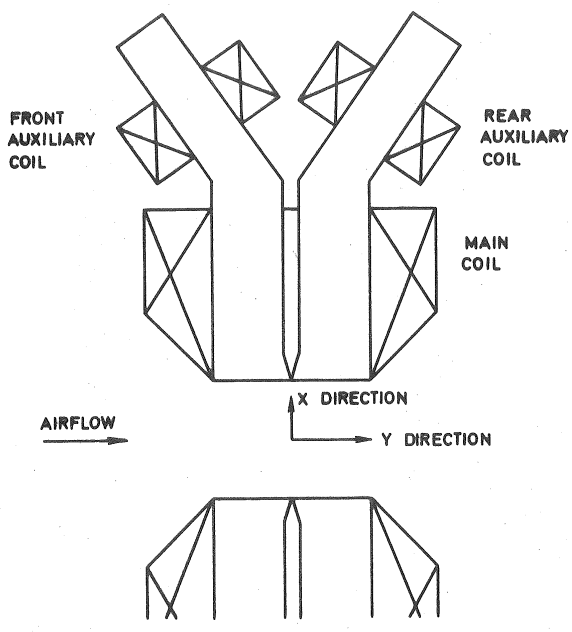

(b) Magnet Arrangement

Fig. 2. Oxford's first MSBS, a two-degree of freedom developed to suspend spherical models in the LDT.

A photograph and schematic of the 2-axis balance developed by Altmann [4, 10], and as used by Hadjimichalis [11], is shown in Figure 2. The overall layout was in many respects a consequence of the requirement that the magnets be able to operate in a high-vacuum environment without over-heating. While liquid cooling of the coils was used Altmann decided against a pressurised system, instead allowing the coolant to be exposed to the test section. This necessitated a low-vapour pressure coolant. Noting that the specific heat capacity of most liquids is less than half that of water, the cooling requirement was minimised through the use of iron cores which prevented the magnetising field from being aligned with the air-flow axis. Consequently, Altmann arranged the primary supporting magnets to be vertically symmetric about the equilibrium point, producing an unstable equilibrium along the vertical axis. This symmetric arrangement permitted the magnetic flux density to be maintained constant while allowing the flux gradient, and therefore force on the sphere, to be varied. Lateral movement of the sphere was not controlled but was maintained stable by using thin cores so that the flux density was maximum on the centreline. Conversely, the cores were long in the direction parallel to the flow, producing small field gradients in the absence of flow. With flow over the model, relatively large horizontal gradients were required to control the model. These horizontal gradients are produced by the auxiliary coils indicated in Figure 2b.

Measurement of the horizontal flux gradient, and thus the drag on the sphere, was achieved through the use of four magneto-resistors, connected in a Wheatstone bridge arrangement. The Wheatstone bridge was balanced with the sphere suspended in the absence of air-flow. Each pair of resistors was mounted to the faces of the upper and lower magnet cores using copper plates. These copper plates acted to ensure that each resistor was in thermal equilibrium with the adjacent resistor, while the magnet cores acted as heat sinks, ensuring thermal stability.

The power supply was initially based on a D.C. generator capable of supplying 100 A, however failure of the power transistors in the horizontal current controller during Hadjimichalis [11] tests prompted a change to four heavy duty car accumulators. The magnet controller, which converts the signal voltage from the position control system to 
a current variation in the magnet coils, was a transistor-based switching mode controller operating at approximately $1 \mathrm{kHz}$. Compared with the natural frequency of the suspended sphere, this frequency was sufficiently fast as to allow the non-linear dynamics of the magnet current (with respect to the input voltage) to be ignored, greatly simplifying the magnet transfer function used in the position controller. The use of a switching-mode power supply was unique among MSBS at the time [4].

Two different position monitoring systems were implemented by Altmann. The first was a purely analogue system using four photodiodes arranged as shown in Figure 3 with illumination provided by a projector lamp. The diodes were arranged such that when the sphere was at null suspension point, it cast an equal shadow across each diode. The primary advantages of this system were its simplicity and ease of integration with the analogue control system. Its major disadvantages were that the model holder could not produce a shadow on the photodiodes, a difficulty that was neatly solved by Hadjimichalis [11] using the holder shown in Figure 4, and that the range of motion over which control of the suspended body could be exerted was determined by the relative size of the body and detectors.

In an attempt to overcome these disadvantages, which were particularly limiting for very small spheres $(<1 \mathrm{~mm})$, Altmann developed a second position monitoring system which was inspired by the operation of television cameras. The system consisted of a flying spot scanner, essentially a cathode ray tube which produced a moving spot of light, and a photomultiplier detector. The moving spot of light scanned across the viewing area, dividing it into a series of horizontal lines. The model location was determined by the horizontal line at detection and a distance along this line. The flying spot operated at a frame rate of $100 \mathrm{~Hz}$ and consisted of 200 lines per frame. By scanning the field of view from top to bottom, and arranging the circuitry so that the model position was detected only once per frame, Altmann was able to develop a system that was unaffected by the model size or the presence of any mounting hardware below the model and was capable of detecting large model movements. Unfortunately, the light intensity generated by the flying spot scanner was so small that the system could only be operated in a darkened room [4, Chapter 7]. In combination with the increased complexity of the system and circuitry required to create the control signal, this issue prevented the flying spot scanner from ever being put into regular service. Hadjimichalis [11] used the simpler analogue system for his sphere drag study and linear analogue systems were implemented for the new balance developed by Haslam-Jones [1].

\section{B. Experimental Studies}

Operated from approximately 1970 through to 1975, the primary experimental campaign completed with Oxford's first MSBS was that by Hadjimichalis [11] who investigated the effect of wall temperature on the drag coefficient of a sphere across a range of conditions, from the continuum regime through to free-molecular flow. Three different spheres with diameters $1.5875(25) \mathrm{mm}(1 / 16 \mathrm{in}),. 3.1750(25) \mathrm{mm}(1 / 8 \mathrm{in}$.) and $6.3500(25) \mathrm{mm}(1 / 4 \mathrm{in}$.) were tested. Sharp edge orifices were used to expand the test gas from the stagnation chamber. Orifices were chosen because they produce higher Mach number conditions more easily than compared with contoured nozzles, albeit at a cost of flow uniformity. Unlike 
previous experiments in the LDT, Hadjimichalis heated the stagnation chamber up to $600 \mathrm{~K}$ which in combination with liquid cooling of the model spheres, permitted wall-to-total temperature ratios as low as 0.25 to be tested. The data set produced by Hadjimichalis spanned Mach numbers from 6 to 12, post-bow-shock Reynolds $\left(R e_{2}\right)$ numbers from 0.2 to 80, wall-to-total temperature ratios from 0.25 to 1 and freestream Knudsen numbers from 0.03 to 10. An annotated photograph of the experimental set-up is provided in Figure 5 and clearly shows the advantage of the large test section of the LDT.

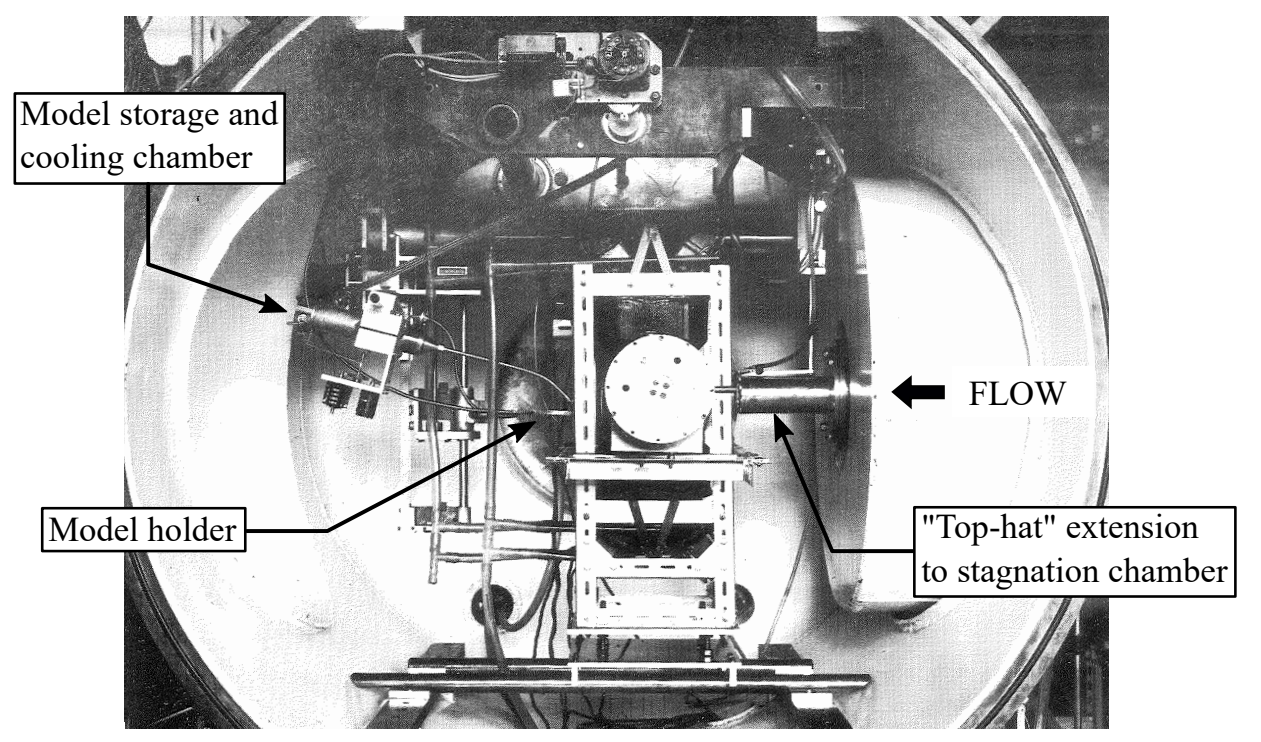

Fig. 5. Photograph of the experimental set-up of Hadjimichalis, including Oxford's first generation MSBS. Adapted from Hadjimichalis [11].

Although, the testing was not without difficulties. Cooling of the magnet coils with an unpressurised system proved inefficient, limiting the total MSBS run time to approximately $20 \mathrm{~s}$. A flow diverter, aka chopper plate, was used to deflect the flow away from the model prior to a test which allowed the facility to run continuously and for the stagnation chamber and pumping system to reach equilibrium. However, Hadjimichalis found that when the flow diverter was removed, the rapid onset of flow over a suspended model was too fast for the balance to respond to, resulting in models being pushed downstream and lost. Overcoming this limitation required manual coordination of the model holder (position), flow diverter and magnet controllers, while keeping within the $20 \mathrm{~s}$ time limit of the magnets. The model cooling box unit was designed to store multiple spheres, thereby reducing the number of times the test section had to be vented back to atmosphere in any given run.

Despite these difficulties, the data obtained by Hadjimichalis was excellent, with a reported (maximum) uncertainty in drag coefficient of $2.6 \%$. Figure 6 presents the results as the variation in drag coefficient with Reynolds number downstream of the sphere bow shock for each of the wall-to-total temperature ratios tested. Over the range $1 \leq R e_{2} \leq 100$ the experimental results compare very well with the theoretical model (labelled 'transition flow model') which used modified Newtonian theory for the pressure drag and the work of Liu [17], Pavlov [18] and Wainwright [19] for the calculation of viscous drag. The work of both Altmann and Hadjimichalis demonstrated the suitability of using MSBSs to accurately measure aerodynamic forces on small models in rarefied hypersonic flows but was limited by the fact that the balance developed only controlled lift and drag. It was suitable for investigating spheres however there was an increasing requirement to characterise more complex geometries such as cones under similar flow conditions. This limitation would be addressed by Haslam-Jones [1] in the late 1970s.

\section{The Redesign, late 1970s through 1980s}

\section{A. Balance Description}

Following the success of Oxford's first two-axis MSBS, a second system was developed during the mid-to-late 1970s by Haslam-Jones [1]. Much like his predecessor Altmann, Haslam-Jones undertook the development as a part 


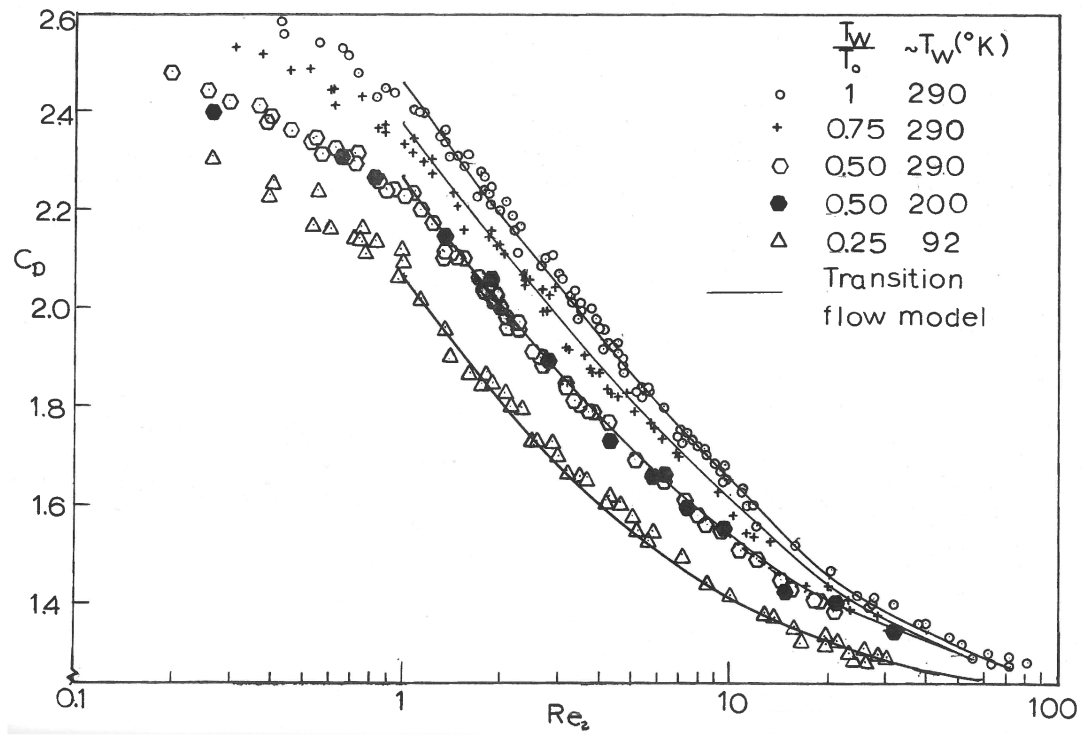

Fig. 6. Variation in sphere drag coefficient with post-bow shock Reynolds number for different wall-to-total temperature ratios. Reproduced from Hadjimichalis [11].

of his doctoral studies and again the system was designed specifically for the LDT. The purpose of the new balance was to enable accurate measurement of the aerodynamics of cone models in the transitional flow regime (between free-molecular flow and continuum flow) where theoretical treatments of the merged shock-boundary layer are difficult to obtain. The increased aerodynamic complexity of a cone (compared with a sphere) required a balance capable of actively controlling the pitching, in addition to the vertical (lift) and horizontal (drag) displacements. Rolling was neglected and, like Altmann's two-axis MSBS, lateral movement and yaw were not controlled but were designed to be stable. Ferro-magnetic cores were again necessary to reduce the cooling requirement and, to improve the linearity of the system, were arranged in symmetric push-pull pairs.

A schematic of the balance designed by Haslam-Jones [1] is shown in Figure 7. It consists of symmetric horseshoe shaped cores in the vertical plane, each with a single lift coil on the base and pitch coils on each leg. In the centre, perpendicular to both the flow direction and the plane of the horseshoe cores, are two air-cored coils which magnetise the model and enable control and measurement of drag. The centre of these coils is $120 \mathrm{~mm}$ square and defines the test cell. The minimum size of this test cell was dictated by the LDT Mach 6 nozzle exit diameter $(110 \mathrm{~mm})$. The lift coils are operated with opposite polarity currents such that their fields cancel at the centreline of the test cell. Combined with the field produced by the drag coils, this produces a vertical gradient of the axial flux density, balancing the model weight. The two air-cored coils are located upstream and downstream (respectively) of the horseshoe magnet suspension point. Altering the current in each at fixed total current (across the two) produces a horizontal gradient of the axial flux density, thus providing control and measurement of drag [1, 12]. The four horseshoe leg coils produce a uniform vertical field which enables control of the model pitch. For reasons of simplicity, lateral stability was again desired but is not naturally achieved by the arrangement in Figure 7 since the field strength is a minimum at the centre of the air-cored coils. Haslam-Jones solved this by placing ferro-magnetic strips on the inside lateral edges of the air-cored coils, absorbing the magnetic field and creating a local maximum along the centreline.

Unlike Altmann [4], Haslam-Jones did not design the horseshoe cores and coil system using a theoretical approach, but instead developed it experimentally via iteration using simplified "half-scale" mild steel cores, sparsely wound cooper wire coils and measurement of the magnetic field using a Bell Gaussmeter. Given the computing resources available in the early-1970s and that the capability of the first generation MSBS exceeded theoretical predictions by $60 \%$ [4], an experimental approach for the development of the core and coil layout was considered simpler and more accurate than a theoretical approach. With modern computing resources this is likely no longer the case [20,21]. The drag coils were sized theoretically based on experiments with the full-scale horseshoe cores. Calibration of the drag coils related the required current in the coils to a known load applied to a suspended model. Although typical for MSBSs [22-24], this relation is in contrast with the method used on the first generation balance whereby the magnetic flux gradient 
was independently measured and calibrated [4]. It is unclear which method is superior. Further discussion of the drag calibration is given in Section V in the context of Owen's [14] improvements. The maximum total current for the drag coils was set at $40 \mathrm{~A}$. For the lift and pitch coils the maximum design currents were $10 \mathrm{~A}$ and $\pm 25 \mathrm{~A}$ respectively. DC power amplifiers were used rather than switching mode amplifiers as for the first generation balance. This change was necessary because of increased inductive coupling in the balance [1, Section 4.1.2].

One significant improvement of this balance was the new coil cooling system. Noting the practical difficulties encountered by both Altmann [4] and Hadjimichalis [11] due to the limited run-time of the first MSBS, HaslamJones wanted the new balance to be capable of operating for several hours and so match the run-time of the LDT facilty. This was achieved by enclosing each coil in a sealed casing made from either machined perspex (drag coils) or cast Araldite C219 (lift and pitch coils) rather than fibreglass as the first balance casings were made from. The new casings were capable of withstanding a pressure difference of 2 bar, allowing a closed-circuit water cooling system to be used. The increased cooling efficiency was proven during both Haslam-Jones' [1] and Dahlen's [12] test campaigns (Section IV.B) where the new balance was operated continuously in the LDT test chamber for periods of $>1.5 \mathrm{~h}$.

The MSBS model attitude detection system and power supply were also redeveloped for the new balance, though each was inspired by the success of Altmann's [4, 10] designs. The model attitude detection system is shown in Figure 8. This design assumed that conic geometries would be used exclusively, a restriction that was later relaxed by Owen [14] (Section V) and was based on the linear system used for the first generation MSBS, essentially relegating Altmann's complex scanning detection system to history. The new system consisted of four phototransitors mounted on sliding bars, thus allowing the position of each sensor to be adjusted to match the size of the model being tested. A fifth phototransistor was used in conjuction with a

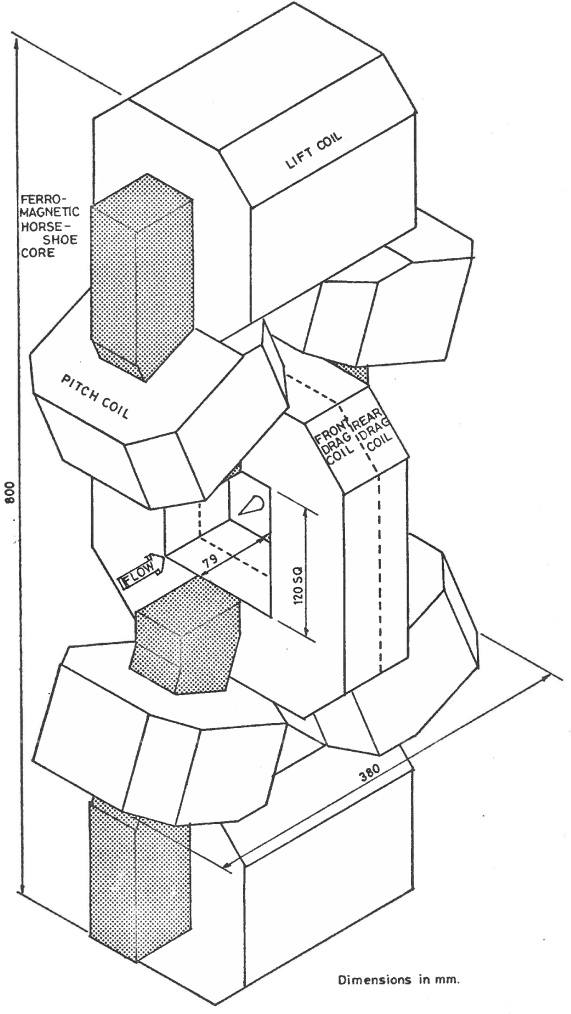

Fig. 7. Oxford's second MSBS, a threedegree of freedom system. shutter to provide a reference signal for axial position control and to monitor the light source. A loss of illumination acted as a interlock for the magnet controllers, turning off power to the coils. Haslam-Jones used the system of mirrors shown in Figure $8 \mathrm{~b}$ to both illuminate the model and reflect the resulting shadow onto the phototransitor assembly. A tungstan-halogen light bulb housed in a water-cooled case provided the necessary illumination.

While the application of digital controllers to MSBSs was beginning to be investigated elsewhere [25], Haslam-Jones achieved active control through the time-tested use of analogue hardware. Separate control loops were implemented for each axis (lift, drag, pitching moment) based on Proportional plus Integral plus filtered Derivative control (PID). The block diagrams are provided in Figure 9. The control hardware was designed and built in-house by the Design Electronics Workshop of the University's Department of Engineering Science. Gains were set by potentiometers and the integral term was only enabled when the model was not physically constrained. Safety interlocks cut power to the coils in the event of failure of the water cooling system, light source or excessive current to the coils. The approximate magnitudes for the control variables that produced stable control were calculated using an analogue computer circuit and an EMIAC II computer unit. Although approximate, this provided sufficient information for the design of the electronic control circuitry. Determination of the actual values needed to produce stable control was undertaken on the physical system during a "protracted development period" [1, pg. 48]. Nevertheless, stable control of cones with half-angles $3^{\circ}$, $5^{\circ}, 10^{\circ}$ and $15^{\circ}$ and lengths ranging from $9 \mathrm{~mm}$ to $53 \mathrm{~mm}$ was achieved.

Following it's development, the new three-component MSBS was put into service and operated without major fault over the next 25 years. Section IV.B presents the experimental results of Haslam-Jones [1], Dahlen [12] and Smith and Lord [13] while Section V discusses the small but important improvements made by Owen [14] and his subsequent 


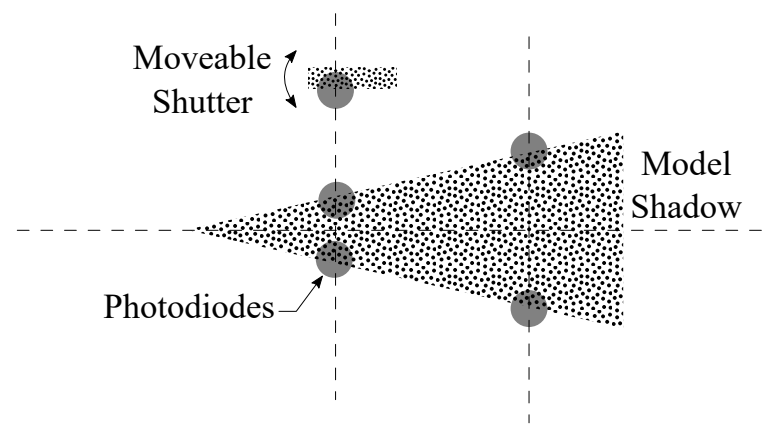

(a) Phototransistor layout

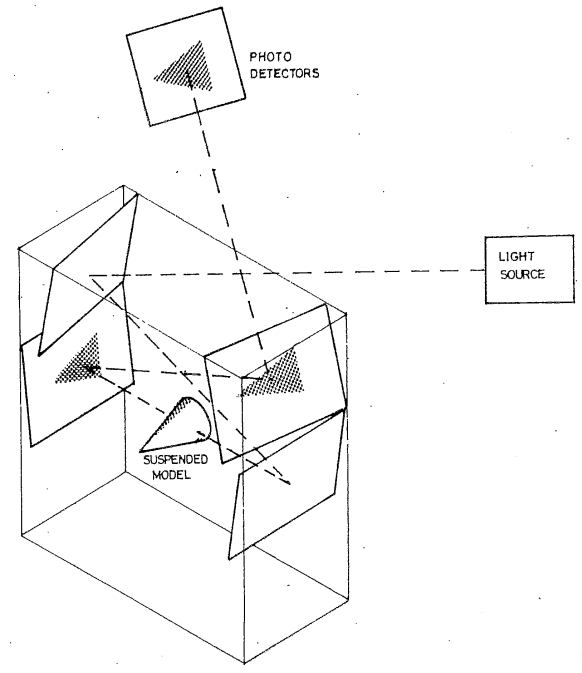

(b) Illumination and detection layout

Fig. 8. Model position detection system developed for Oxford's second MSBS. Redrawn from Haslam-Jones [1].

experimental investigations.

\section{B. Experimental Studies}

The capability of the new balance was demonstrated over the course of three experimental campaigns: firstly Haslam-Jones [1] measured drag on adiabatic sharp cones, then Dahlen [12] investigated the effects of nose bluntness and wall-to-total temperature ratio in an extensive test campaign, and finally Smith and Lord [13] attempted to measure both lift and drag at angle of attack (AOA). Table 1 summarises the different cone geometries tested by each experimenter. Haslam-Jones tested 7 cones, Dahlen a total of 21 and Smith and Lord a single geometry. In all cases the models were made from either mild steel, high carbon steel or fine grained cast iron with Dahlen [12, pg. 27] finding cast iron to be most effective when suspending geometries with small $L / D$ (and hence small axial magnetization vector). Figure 10 shows the second generation MSBS in the LDT test section and a suspended cone.

Figure 11 presents the results of Haslam-Jones as the variation in normalised drag coefficient with Knudsen number where the normalisation is with respect to the free-molecular drag coefficient $C_{D_{f m}}$ which was calculated using the work of Ashley [26]. All data are for cones at $0^{\circ} \mathrm{AOA}$ and ambient wall temperature (i.e. $T_{w} / T_{o} \approx 1$ ). The maximum uncertainty in drag coefficient (including calibration uncertainty) was reported to be just $2 \%$, a slight improvement over that achieved by Hadjimichalis [11] using the first generation balance. The test gas was dry ambient air $\left(T_{o} \approx 296 \mathrm{~K}\right)$ and test conditions were produced using either the Mach 6 contoured nozzle or an orifice. Two different orifice sizes used, a $10 \mathrm{~mm}$ diameter orifice which produced Mach 6 flow and a $6 \mathrm{~mm}$ diameter orifice which produced Mach 9 flow. Reynolds number based on base diameter $\left(R e_{\infty, d}\right)$ covered the range 119 - 1224. Flow divergence within the free-jet from the orifice (compared with parallel flow from the contoured nozzle) was taken into account by calculating flow conditions at the half-area plane ${ }^{\ddagger}$ which produced good agreement with data from the contoured nozzle. Test conditions were calculated using isentropic flow theory with Mach number calculated from experimental Pitot pressure measurements for the contoured nozzle and from the work of Ashkenas and Sherman [27] for the free-jets. The viscosity was calculated using the Chapman-Enskog formulation, with values for the Lennard-Jones potential taken from Monchick and Mason [28].

The data in Figure 11 show the expected trend of increasing drag coefficient with Knudsen number although results for the slender $3^{\circ}$ and $5.4^{\circ}$ cones lie distinctly below the data for the fatter cones. Haslam-Jones [1, pg. 86] suggested this was because slender cones feature a longer merged viscous layer and velocity slip at the wall within this layer would reduce the viscous drag. However, it may simply be an artefact of normalisation by the free-molecular drag coefficient which increases significantly for slender cones at Mach numbers less than 10 because random molecular motion causes additional surface collisions than is expected based on the frontal area. Haslam-Jones made an attempt at correlating all

${ }^{\ddagger}$ Defined as the cross-sectional plane for which there is an equal wetted area upstream and downstream of the plane. 


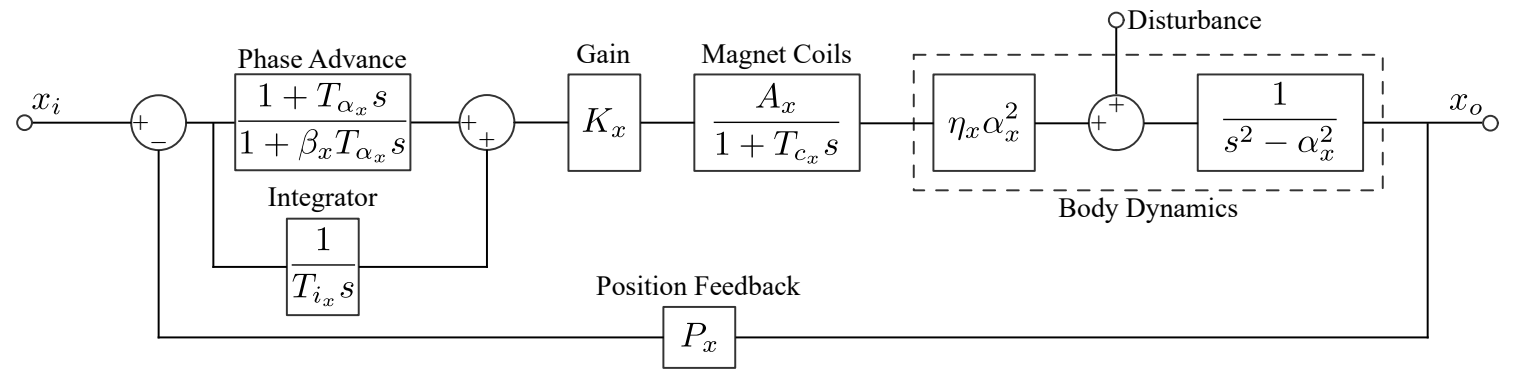

(a) Drag

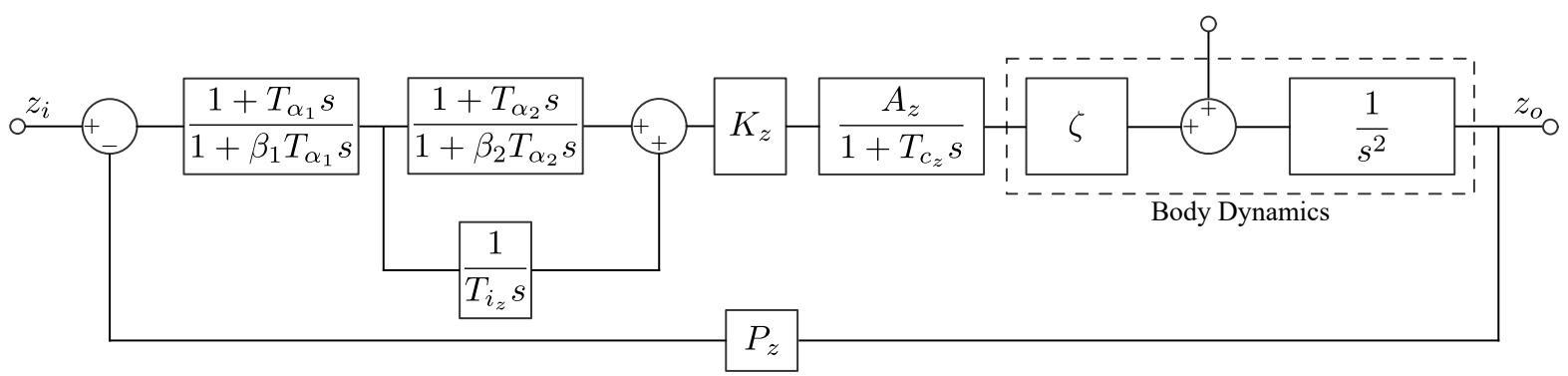

(b) Lift

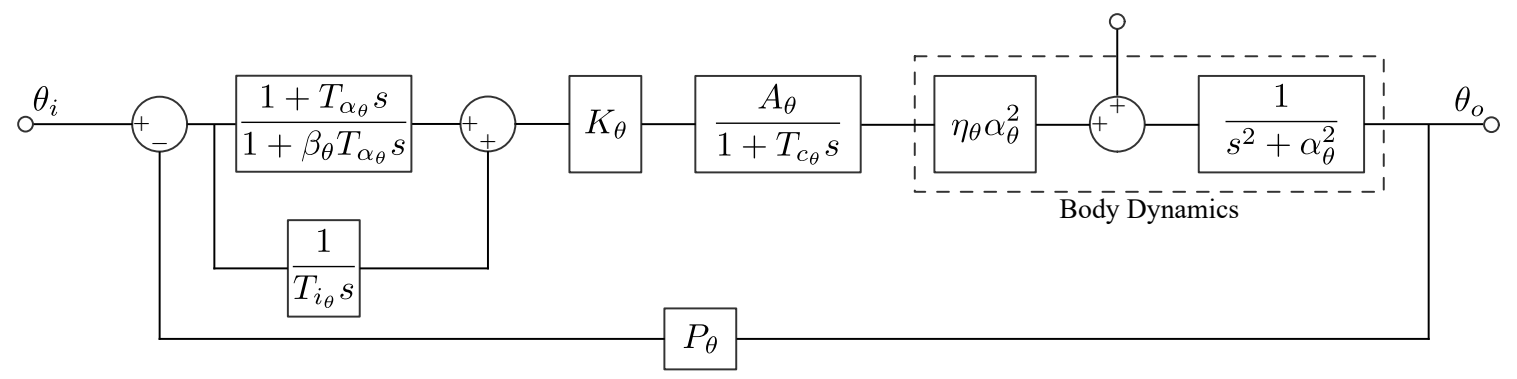

(c) Pitch

Fig. 9. Control block diagrams for the three-component MSBS of Haslam-Jones [1] (redrawn).

Table 1. Summary of Nominal Cone Dimensions

\begin{tabular}{lccc}
\hline Experimenter & Half-Angle, $^{\circ}$ & Base Diameter, mm & Nose Bluntness Ratio, $r_{n} / r_{b}$ \\
\hline Haslam-Jones [1] & 3 & 5 & 0.02 \\
& $5,10,15$ & 5,10 & “ \\
\hline Dahlen [12] & 3 & 2.5 & $0,0.3$ \\
& 3 & 5 & $0,0.1,0.2,0.3,0.4$ \\
& 6 & 5,10 & “ \\
& 10,15 & 5,10 & 0 \\
\hline Smith and Lord [13] & 10 & 10 & $?$ (sharp) \\
\hline
\end{tabular}




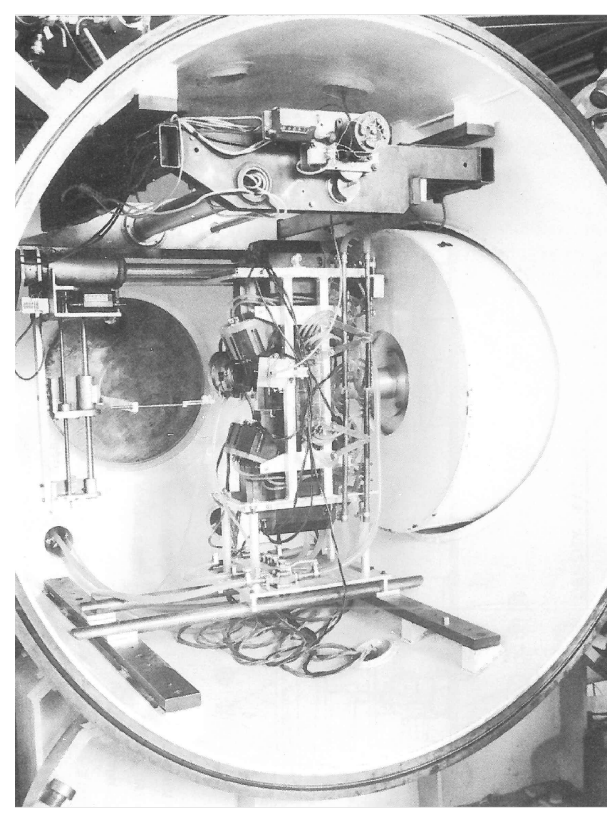

(a) The three-component MSBS in the test section, circa late 1970s. The traversing model holder can be seen on the right.

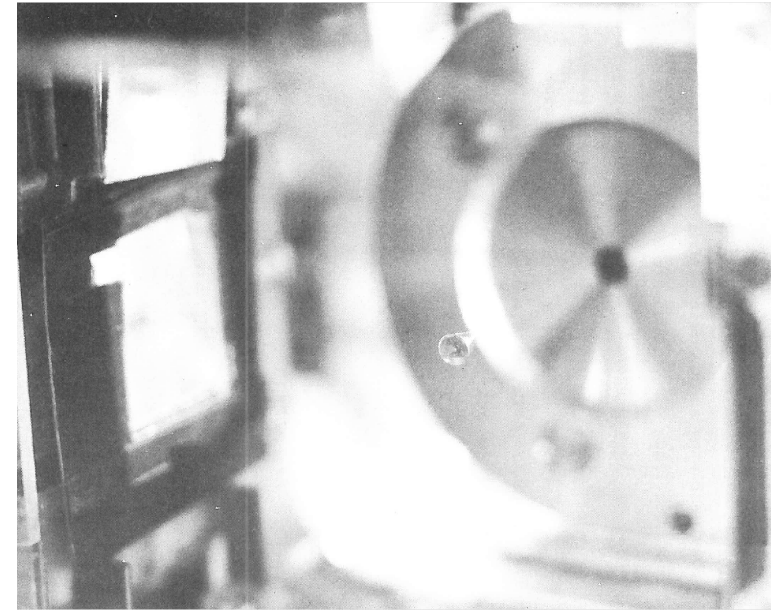

(b) View looking upstream at a suspended cone. Mirrors for the optical detection system can be seen on the left.

Fig. 10. Experimental set up of Haslam-Jones [1] showing the second generation MSBS in the LDT test section and a suspended cone model.

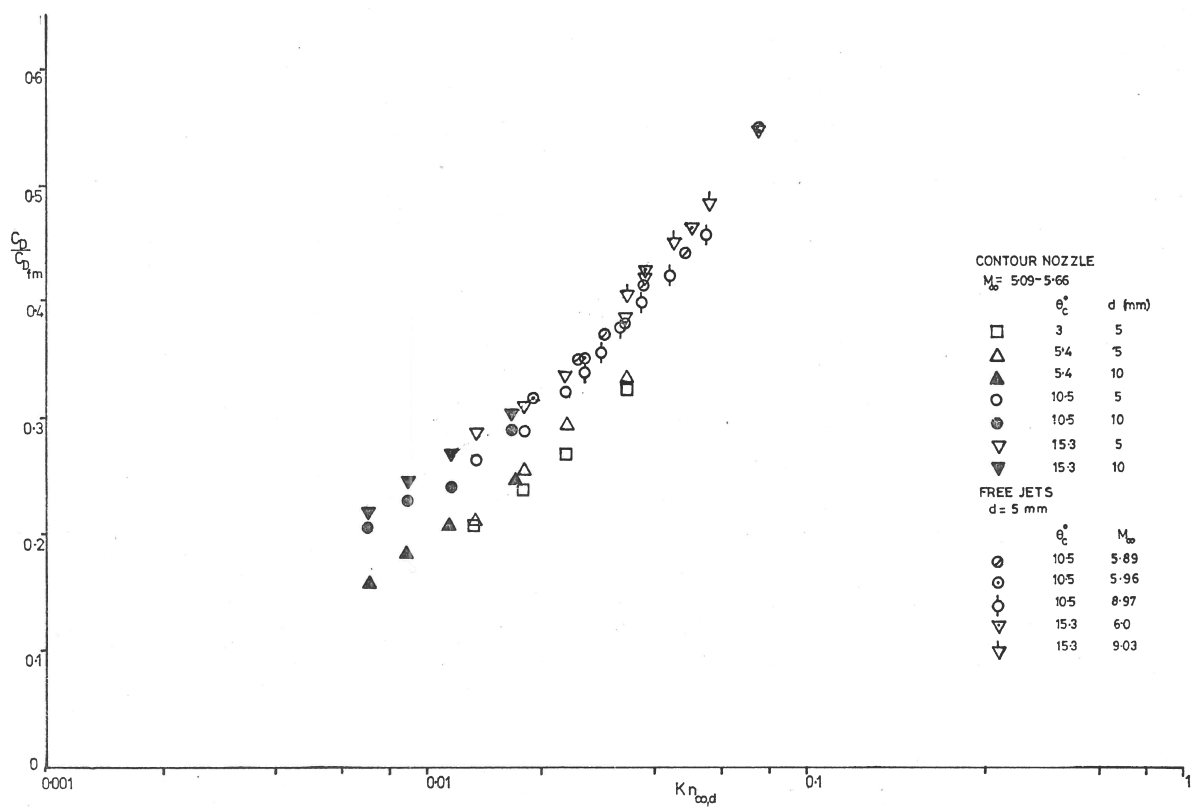

Fig. 11. Variation in cone drag coefficient with freestream Knudsen number. The drag coefficient has been normalised by the free-molecular drag coefficient value for each geometry. Reproduced from Haslam-Jones [1]. 
relevant sharp cone data available in the literature at the time. He found that although his data compared well with other datasets, the overall level of scatter, particularly at high Knudsen numbers could not be completely correlated or explained by the differing experimental methods used across the data set (i.e. free-flight testing, sting/supported model tests, MSBS tests). The parameter $\left(C_{D}-C_{D_{i}}\right) /\left(C_{D_{f m}}-C_{D_{i}}\right)$, where $C_{D_{i}}$ is the inviscid cone drag coefficient [29], collapsed data for different cone angles and Knudsen number was found to collapse data across the Mach number range but did not correlate hot and cold wall data. Other difficulties encountered when assembling the dataset were ensuring that a consistent definition of gas viscosity was used and calculating the viscosity at the very low freestream temperatures $\left(T_{\infty}<100 \mathrm{~K}\right)$ present in the tests. Haslam-Jones [1] ultimately concluded that deeper understanding of cone drag in the rarefied transition regime would require "a large volume of work to be carried out with the MSBS," a challenge that Dhalen would take up.

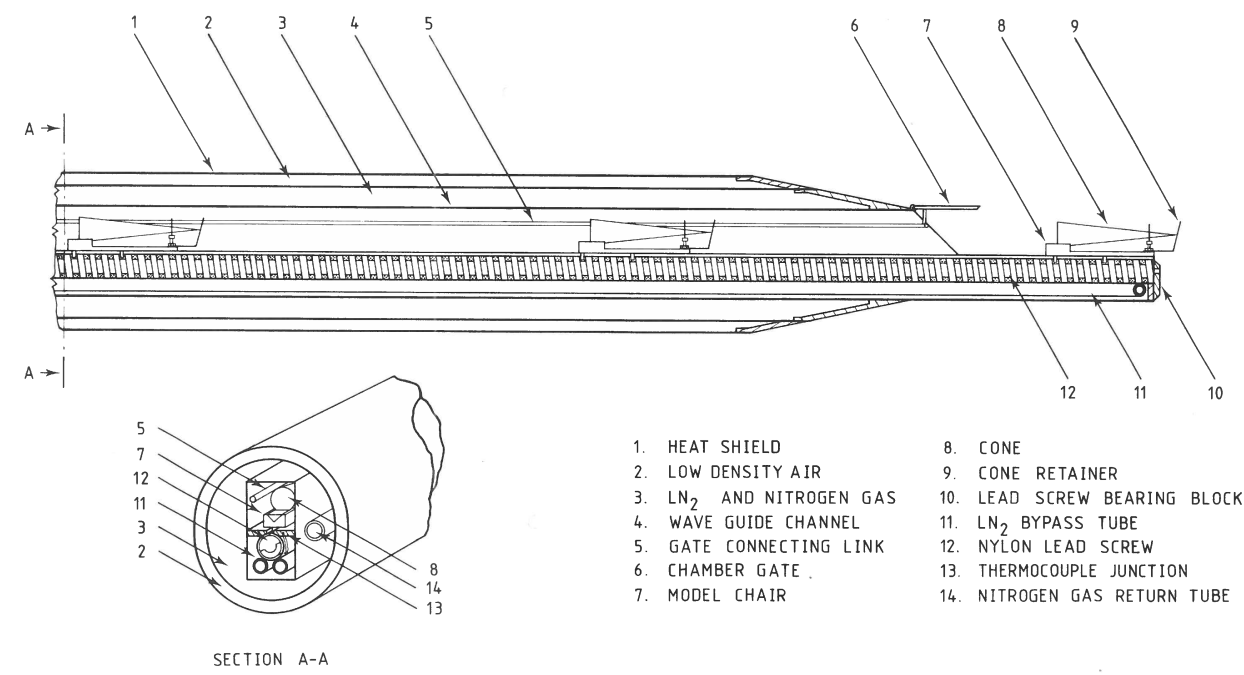

Fig. 12. Liquid nitrogen cooled model holder developed by Dahlen [12] for conical models.

Following Haslam-Jones' success, Dahlen [12] completed an extensive study of cone drag focussing on the effects of wall-to-total temperature ratio $\left(T_{w} / T_{o}\right)$ and nose bluntness ratio $\left(r_{n} / r_{b}\right)$. In total 21 geometries were tested (Table 1) covering wall-to-total temperature ratios from 0.17 to 1.0 , Reynolds numbers $\left(R e_{\infty, d}\right)$ from 50 to 1300 and Mach numbers from 5.2 to 5.9. The Mach number variation was small because Dahlen did not use orifices free-jets, instead solely relying on the LDT Mach 6 contoured nozzle to expand the test gas. Test conditions were calculated using the same methodology as Haslam-Jones [1] but with values for the Lennard-Jones potential taken from Hirschfelder et al. [30]. The uncertainty in measured drag coefficient was in the range 0.94 to $1.90 \%$ depending on the cone size. As a part of his study Dahlen redesigned the nozzle stagnation chamber to improve heating of the inflow gas and, similar to Hadjimichalis, developed the holder shown in Figure 12 to cool and dispense models. The new stagnation chamber featured a bypass nozzle that allowed steady mass flow rate (and therefore steady stagnation temperature and pressure) to be established prior to initialisation of flow over the model via manual linear valves which closed the bypass nozzle and opened the primary nozzle (and vice versa). Compared with the flow diverter plate used by Hadjimichalis (see Section III.B) this system reduced the thermal load on the MSBS and allowed the model temperature to be accurately calculated [12, Appendix A.2.]. The ability of the second generation MSBS to maintain control and suspension of a model during the dynamic loading associated with flow initialisation and to operate for extended periods under vacuum were significant improvements over the first generation MSBS.

Figure 13 presents the results of Dahlen's experimental investigation into the effect of wall-to-total temperature ratio. The results are plotted as the variation in drag coefficient with Knudsen number with each sub-figure representing a single cone half-angle. Data at wall-to-total temperature ratios of 1.0, 0.75 and 0.23 were obtained using ambient temperature models (and heated inflow gas) whereas data at temperature ratios of 0.56 and 0.17 were obtained by cooling the model to $T_{w}<97 \mathrm{~K}$. The typical uncertainty, in both drag coefficient and Knudsen number, is indicated in Figure 13a. At elevated stagnation temperatures, Dahlen noted a discrepancy between the temperature measured using a thermocouple in the stagnation chamber and that inferred from mass flow rate and stagnation pressure measurements. 


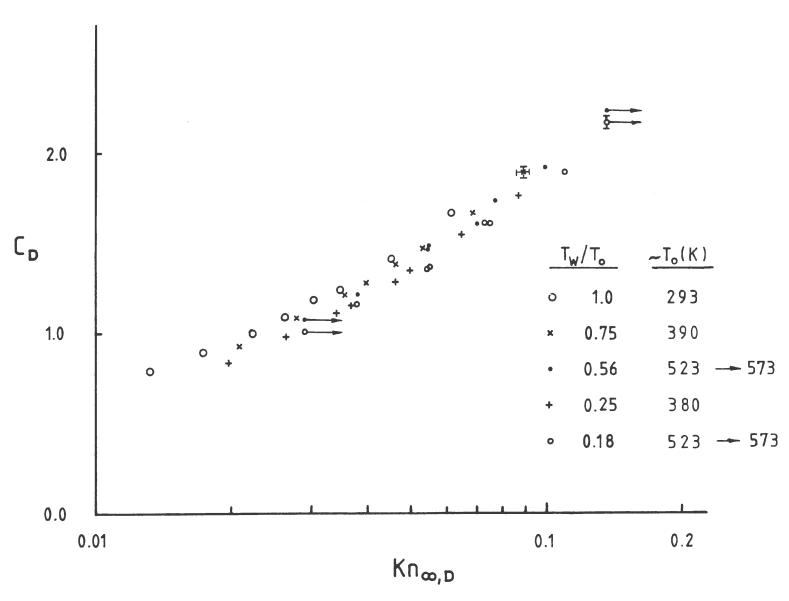

(a) $3^{\circ}$ cone

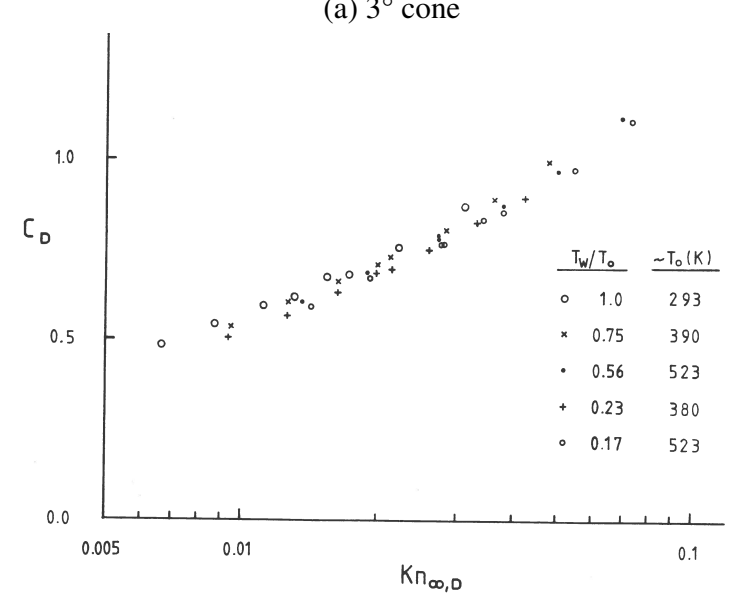

(c) $10^{\circ}$ cone

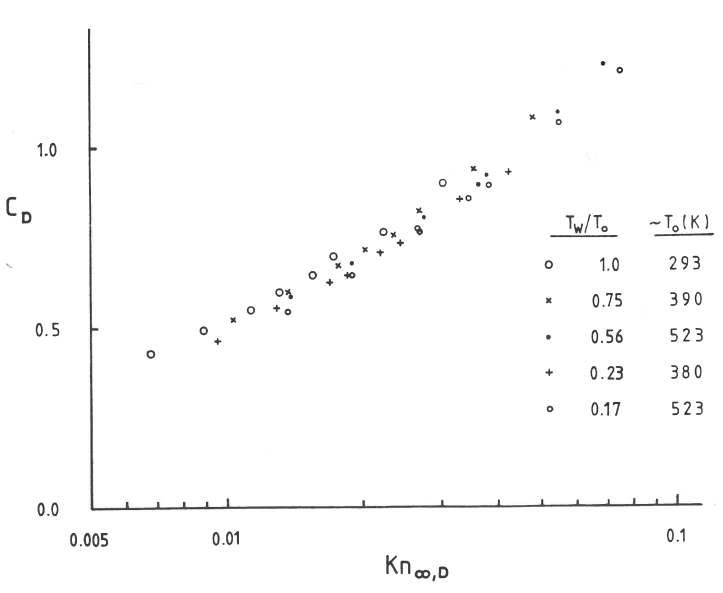

(b) $6^{\circ}$ cone

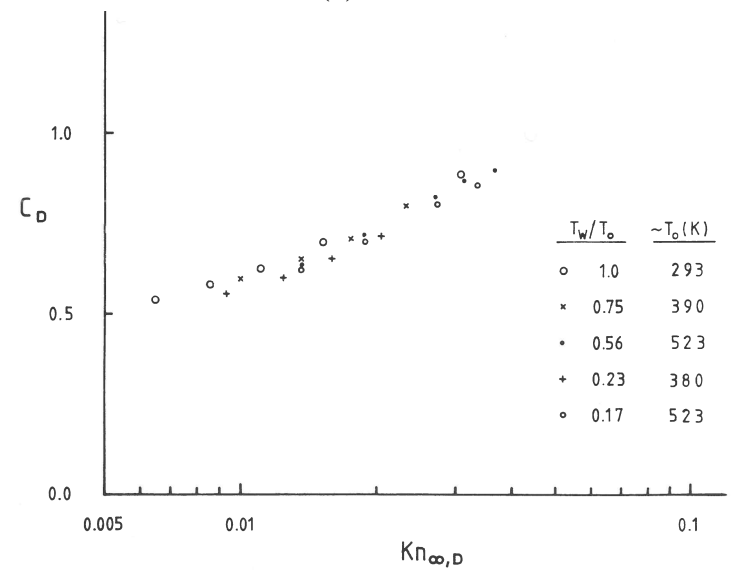

(d) $15^{\circ}$ cone

Fig. 13. Drag coefficient variation with Knudsen number for different (sharp) cone half-angles and wall-to-total temperature ratios. Reproduced from Dahlen [12].

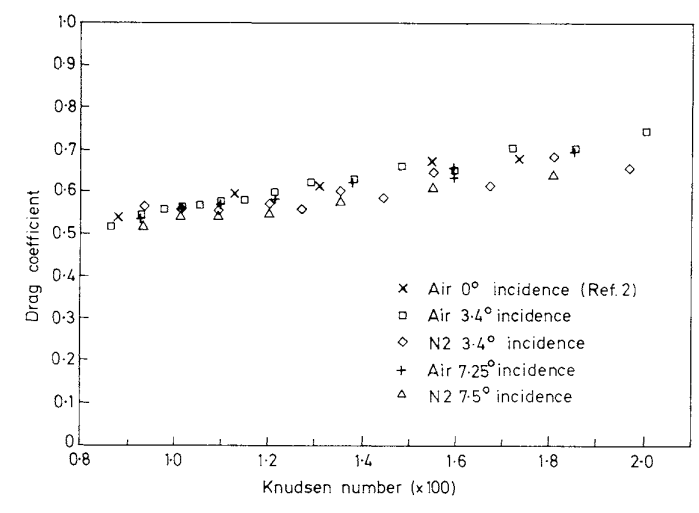

(a) Drag Coefficient. Ref. 2 mentioned in the legend is Dahlen [12].

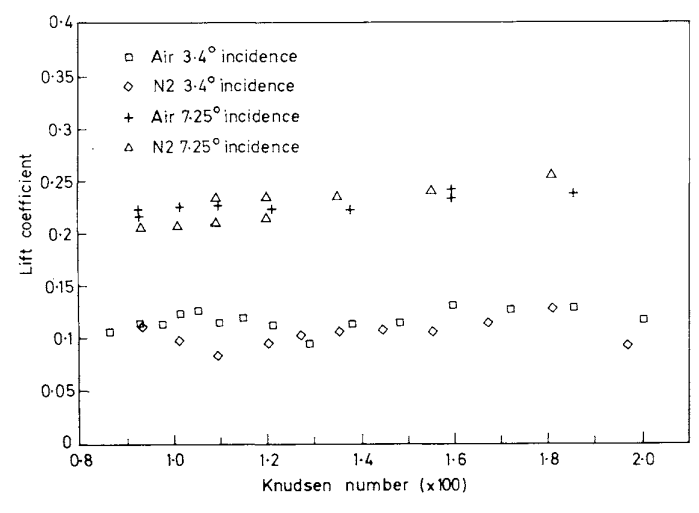

(b) Lift Coefficient

Fig. 14. Variation in lift and drag coefficient with Knudsen number for a $10^{\circ}$ half-angle cone at various angles of attack. 
The temperature from the later calculation was used for data reduction; the potential influence of the discrepancy is indicated in Figure 13a (for $T_{w}=523 \mathrm{~K}$ ). Analysis by Dahlen of the data plotted in Figure 13 led to the following conclusions: (1) reducing the wall temperature ratio at fixed Knudsen number decreases the drag coeffient; (2) the effect of reducing the wall temperature ratio on drag is relatively larger for slender cones than for fatter cones; (3) decreasing the wall temperature from $T_{w} \approx T_{o}$ to $T_{\infty}$ decreases the drag by less than $10 \%$; (4) over the range of conditions used, increasing rarefaction reduces the influence that changing the wall temperature ratio has on the drag coefficient. Dahlen made various attempts at correlating the set of data and also undertook extensive comparisons with data sets (including Haslam-Jones) available from the literature and with available theoretical predictions for near-continuum and near-free molecular flows. Readers are referred to Dahlen [12] for full details which are too extensive to be discussed here. Comparisons of the blunt cone data with DSMC simulations are reported in Dahlen et al. [31] and good agreement was found. Overall Dahlen found that no single correlation or theory collapsed the data and that large scatter existed between the various data sets.

Although the second generation balance controlled three degrees of freedom (lift, drag, pitch) and so was theoretically capable of measuring three-components of force, both Haslam-Jones and Dahlen focussed only on quantifying drag for cones at $0^{\circ} \mathrm{AOA}$. In an effort to extend the balance capability, Smith and Lord [13] attempted to measure threecomponents of force on a cone at angle of attack. A single cone with half-angle $10^{\circ}$ and base diameter $10 \mathrm{~mm}$ was tested using the Mach 6 contoured nozzle at angles of attack of $3.4^{\circ}$ and $7.5^{\circ}$. The results for lift and drag coefficient are given in Figure 14. When models are at $0^{\circ}$ angle of attack, the control circuits for lift, drag and pitching moment are completely independent. This independence disappears for finite angles of attack because the model detection system (Figure 8a) must be rotated so that it is aligned with the model axis. The resulting signals from the detection system are in the body-fixed coordinate system, coupling the lift, drag and pitching moment coil signals. Coupling is also introduced because the magnetization vector is no longer solely aligned with the drag coils. Smith and Lord found that the coupling did not produce strong non-linearities into the calibration, making it possible to measure all three aerodynamic force components.

Figure 14 presents the lift and drag coefficient data measured by Smith and Lord. The results show that over the range of conditions tested, the angle of attack does not have a strong influence on the drag. Smith and Lord note that this is because the angles tested are less than the cone half-angle. In comparison, lift coefficient varies approximately proportionally with angle of attack. Smith and Lord did not present any results for pitching moment because the resultant line-of-action was less than $2 \mathrm{~mm}$ from the centre-of-mass across all tests conducted. Nevertheless, Smith and Lord's investigation showed the second generation MSBS was capable of controlling and measuring multiple components of force on models in rarefied flow conditions.

\section{Refinement, 1990s through 2000s}

\section{A. Balance Description}

Owen [14] was the last person to use the MSBS and, as in the earlier studies, it was a key piece of experimental infrastructure that enabled his investigation into the rarefied aerothermodynamics of simple re-entry vehicle geometries. The overall structure of the three-component MSBS and its control system had remained unchanged since Haslam-Jones [1] finalised the design in the late 1970s and would remain unchanged throughout Owen's work and up to current times. Nevertheless, Owen made three significant improvements to the MSBS sub-systems and experimental process including (1) a new calibration procedure that gave superior accuracy, particularly for very small model sizes, (2) a new optical tracking system that removed the minimum model size limitation of the previous system and allowed non-conical models to be suspended, and (3) a dedicated data acquisition program, written in Microsoft Quickbasic for an IBM 486 computer and Data Translation DT2811 analogue-to-digital board, which synchronised and automated the recording of data from both the MSBS and three-axis traverse used for wake-flow surveys. Although the data acquisition program and three-axis traverse enabled the efficient completion of Owen's experiments, both systems have recently been further upgraded as a part of the refurbishment and recommissioning of the LDT $[15,16]$ and so will not be further discussed here; interested readers are instead referred to Owen [14, Appendix D].

The new optical detection system implemented by Owen is shown in Figure 15. Two important changes were made compared with the previous system (Figure 8a). Firstly, the four individual photoresistor sensors were replaced by a single large photodiode that was subdivided into quadrants and fitted with an optical bandpass filter. Secondly, the tungsten-halogen light source was replaced by a low power $(1 \mathrm{~mW})$ class 3 diode laser operating at a wavelength of $570 \mathrm{~nm}$. The use of a single large photodiode addressed the model size constraint that existed for the four-spot system 


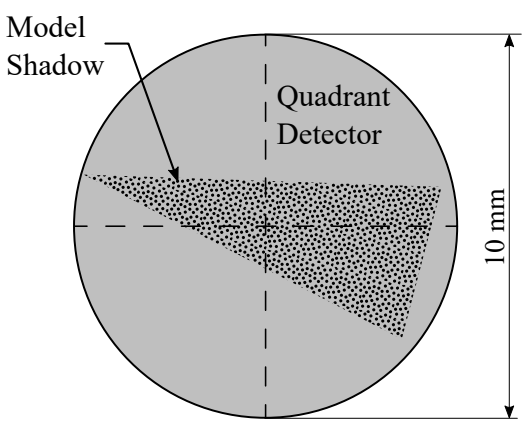

(a) Quadrant photodiode with model at angle of attack.

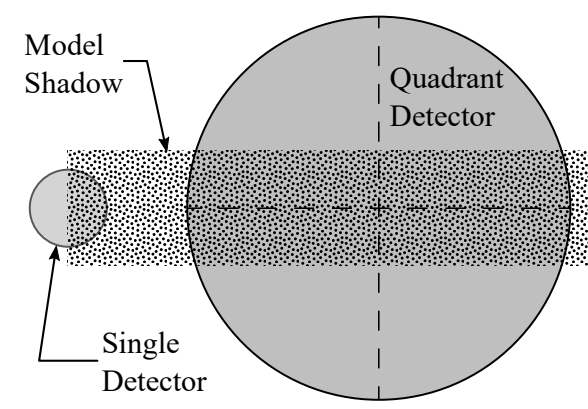

(b) Postion detection for non-conical models.

Fig. 15. The improved optical detection system implemented by Owen [14] (redrawn).

and enabled position detection at a wide range of angle of attack (without requiring manual adjustment of the detector position). Since the quadrants operated in a similar way to the four original detectors, no significant modifications to the control circuits were required. Owen also added an second spot-style photodiode and laser source to monitor the rearmost feature of a model independently of the quadrant sensor, thereby extending the system for use with non-conical geometries (Figure 15b). Furthermore, the introduction of a laser light source, in combination with the bandpass filter, provided stable illumination, removing the need for the fifth monitoring detector of the original system and eliminating the influence of stray light. Overall, the new detection system is both more capable and more reliable than the previous system.

The final improvement that Owen made was not to the MSBS hardware itself but to the calibration procedure. It is well known that calibration of conventional multi-component (strain-gauge) force balances is difficult due to cross-coupling between components. MSBSs are not exempt from these difficulties [22, 32] and can suffer from additional complexities due to non-linearities. Although Smith and Lord [13] had some success with calibrating both the lift and drag components the primary use for the balance has been the measurement and characterisation of drag. The arrangement of the magnets (Figure 7) ensures the drag coils act independent of the lift and pitch coils. The calibration method used by Haslam-Jones [1] consisted of fine thread that was glued to the rear of the model and looped over a pulley such that supported masses would impart a known axial load to the model. Only models larger than $\approx 5 \mathrm{~mm}$ (base diameter) could be calibrated (due to self-weight of the thread affecting model suspension) and uncertainties associated with friction and pendulous motion of the suspended mass limited the accuracy and repeatability. Dahlen improved on Haslam-Jones' method by using a precision microbalance (CI Precision Mark 2B) in combination with a $0.80 \mathrm{~mm}$ diameter aluminium tube to apply calibration loads between approximately 0.8 and $7.0 \mathrm{mN}$ to the tip of a suspended model. The uncertainty in applied load was at most $0.14 \%$. Although an improvement, this calibration

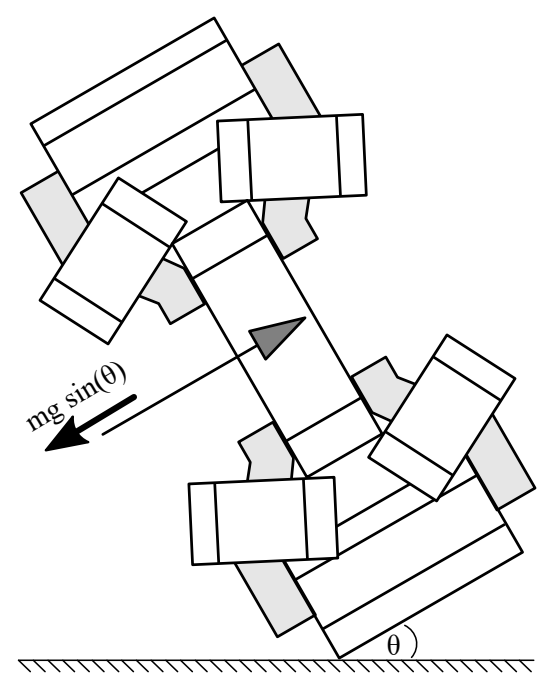

Fig. 16. New drag calibration method of Owen [14] using the model self-weight. method was still quite involved, requiring great care on the part of the experimenter to ensure precise alignment of the aluminium tube with the model. Consequently, Owen developed the alternative method shown in Figure 16 whereby the entire MSBS was tilted off-vertical and the axial component of the model weight was used as the calibration load. By measuring the tilt angle with an inclinometer to a precision of 1 arcminute, the smallest load that can be applied is just $0.03 \%$ of the model mass. In comparison, the smallest calibration load reported by Dahlen was $0.8 \mathrm{mN}$ which represents $\approx 0.8 \%$ of the model mass for a $6^{\circ}$ cone with $10 \mathrm{~mm}$ base diameter [see Fig. 3.6 of 12]. As such, the new calibration method implemented by Owen is easier to undertake, 
allows finer variations in the applied load and has an accuracy limited only by the measurement of inclination angle, model mass and coil current.

\section{B. Experimental Results}

Throughout and following his doctoral studies Owen [14, 33-35] completed a number of experimental studies which demonstrated the capability of the MSBS for enabling accurate characterisation of rarefied aerothermodynamics. Owen's studies included -

1) Measurement of drag coefficient for sharp cones at angles of attack larger than the cone half-angle;

2) Pitot pressure surveys of the wake flow behind cones at angle of attack;

3) A feasibility study on measurement of the heat transfer to suspended cones using liquid crystals;

4) An investigation of the influence of model sting length on drag coefficient and the indirect influence of a nearby probe;

5) Drag measurements of axially aligned cylinders of varying length-to-diameter ratios;

6) Drag measurements of an Aerobrake geometry;

7) A feasibility study of the NASA Hyper- $X$ flight vehicle stage separation manoeuvre.

Only selected results will be presented here. Interested readers are referred to Owen's thesis [14] and conference papers [33-35] for further details.

Test conditions were generated using either the contoured Mach 6 nozzle or a free-jet produced by a Mach 3 conical nozzle. Data reduction was consistent with the methods of Dahlen and Haslam-Jones (as described in Section IV.B). A range of models were used and were machined from either sliver steel or mild steel. The Aerobrake was made from balsa wood with a mild steel insert. The two smallest cones tested were a $3^{\circ}$ cone with base diameter $5 \mathrm{~mm}$ and a $6^{\circ}$ cone with base diameter $2.5 \mathrm{~mm}$. This cone was significantly smaller than anything previously tested with the balance. The ability to test this geometry was a direct consequence of the improvements Owen made to the model detection system.

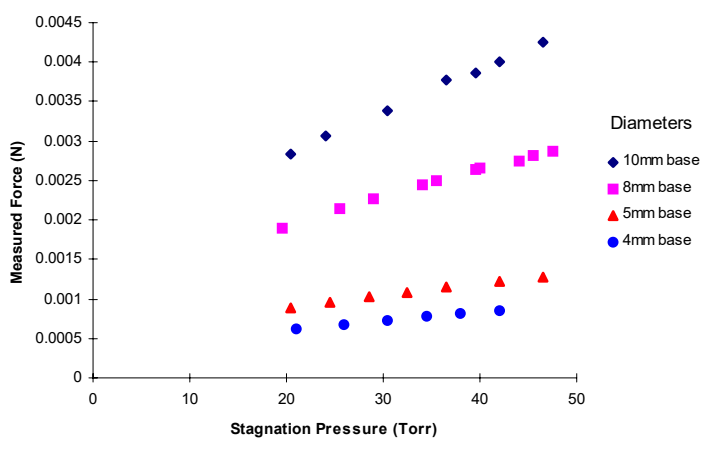

(a) Raw force data ( $0^{\circ}$ angle of attack). 1 torr $=133 \mathrm{~Pa}$

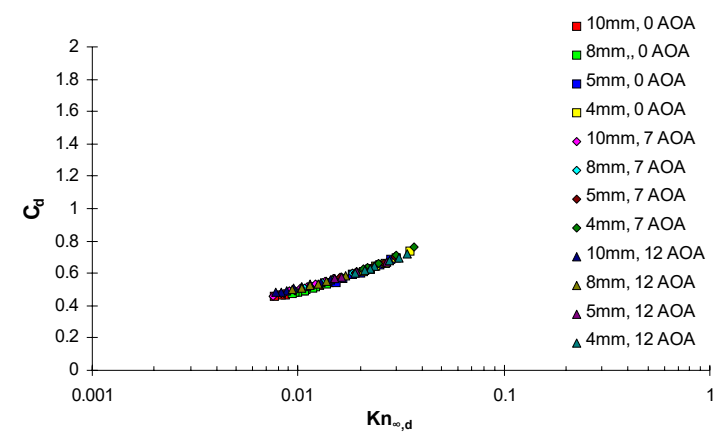

(b) Variation in drag coefficient with Knudsen number for different angles of attack. The drag coefficient has been defined relative to the projected frontal area.

Fig. 17. Experimental results for sharp $6.5^{\circ}$ cones. Reproduced from Owen [14].

Figure 17 presents results for sharp $6.5^{\circ}$ cones. Figure 17a plots the raw measured force against nozzle stagnation pressure, demonstrating that the MSBS is able to resolve sub-milli-Newton forces, a capability that is difficult to achieve using traditional strain-gauge based force balances. Figure 17b plots the variation in drag coefficient with Knudsen number for various angles of attack. The good collapse of the data is a consequence of defining the drag coefficient with respect to the projected frontal area, rather than with respect to the base diameter. Note that the cone was able to be suspended at angles of attack larger than the cone half-angle, a capaibility that was not possible with the original model attitude detection system.

The ability of the balance to suspend non-conical models was demonstrated by Owen through testing of the Aerobrake geometry shown in Figure 18a. The Aerobrake model was made from balsa wood and featured a mild steel afterbody to enable suspension and to maintain compatibility with the attitude detection system. Experimental results obtained for the Aerobrake are compared with the Direct Simulation Monte Carlo (DSMC) calculations of Gilmore et al. [36] in Figure 18b. Excellent agreement was found between the experimental data and simluations and both data sets indicated a small decrease in drag with increasing Knudsen number; a result that is opposite to that observed for slender cones (cf. 


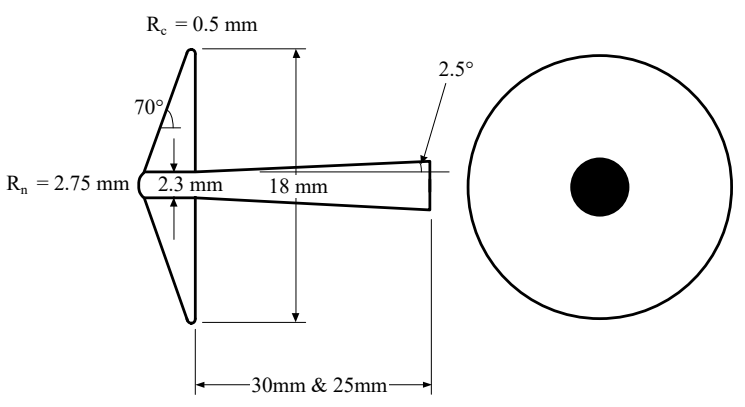

(a) Aerobrake geometry

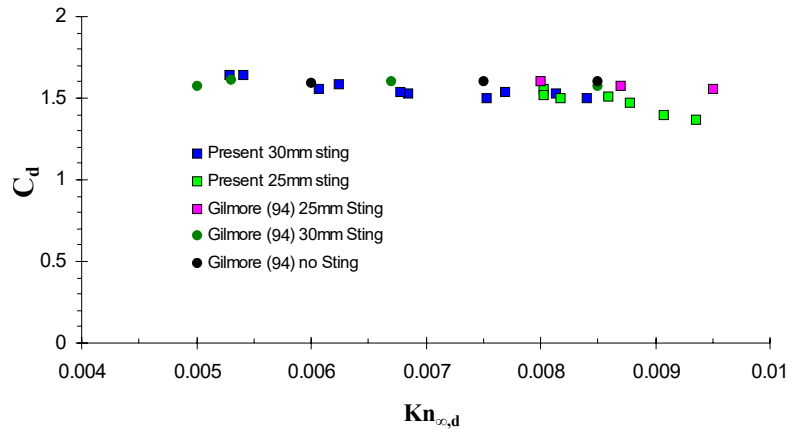

(b) Variation of drag coefficient with Knudsen number

Fig. 18. Aerobrake geometry and drag coefficient results compared with DSMC simulations. Reproduced from Owen [14].

Figures 13 and 17). This behaviour was attributed by Owen to be due to reattachment of recirculating flow in the wake region producing a negative shear component. Further experiments are required to confirm this hypothesis.

Perhaps the most visually striking results produced by Owen are the pitot pressure surveys in the wake flow as reproduced in Figure 19. Figure 19a shows a contour plot of normalised pitot pressure in the horizontal symmetry plane behind a sharp, $6.5^{\circ}$ cone at Mach 5.4 and $K n_{\infty, d}=0.028$. The cone was at $0^{\circ}$ angle of attack. The normalisation was with respect to the freestream pitot pressure and these data were obtained by traversing a $1 \mathrm{~mm}$ diameter pitot probe at increments of $0.2 \mathrm{~mm}$ behind the cone. Measurement at such high spatial resolution was only possible through use of the new traversing mechanism and data acquisition program implemented by Owen. Section V.B presents similar results for a $5 \mathrm{~mm}$ diameter, $5^{\circ}$ blunt cone $\left(r_{n} / r_{d}=0.375\right)$ at angles of attack of $0^{\circ}, 5^{\circ}$ and $10^{\circ}$. The surveyed vertical plane was $0.1 d(0.5 \mathrm{~mm})$ downstream of the base. In all the surveys shown in Figure 19 a deep low pressure region at the centre of the wake is present. This is bounded by a broad region of shocked gas. High quality, undisturbed survey data such as shown in Figure 19 are valuable for simulation validation and are typically very difficult to acquire with traditionally mounted models, particularly in rarefied flows where viscous effects are strong and regions of influence are large.

The final and perhaps most ambitious experiment undertaken by Owen and Owen was in support of the NASA Hyper-X program. The experiment was a part of a small feasibility study to demonstrate the capability of investigating stage separation events in the LDT with the MSBS. Separation of the X-43A scramjet powered flight vehicle from the Pegasus rocket booster was an area of concern for the main program as both geometries were non-axisymmetric and so there was little prior experience or data available [37, Chapter 8]. An extensive research program utilising both experimental data from the NASA Langley and Arnold Engineering Development Complex wind tunnels and state of the CFD simulations was developed to understand the dynamic separation event and ensure it wouldn't result in loss of the experimental vehicle. The project in the LDT did not directly contribute to the Hyper-X program but was instead focused on capability demonstration at rarefied flow conditions. Figure 20 shows photographs of the stage separation under wind-off conditions using the MSBS. The X-43A vehicle and booster were $2 \%$ scale models. The X-43A model was made via stereolithography and featured a metal insert to allow suspension. The booster model was connected to a 3 -axis traverse. The X-43A vehicle geometry is clearly non-symmetric and represented a significant departure from the axisymmetric models that had been tested up to this time. Successful suspension of this geometry shows the progress that had been made since 1977 when Haslam-Jones [1] completed the second generation MSBS. Although successfully suspended under wind-off conditions, the large drag of the X-43A geometry proved difficult to control under wind-on conditions. Unfortunately these difficulties were not solved prior to the end of the short project and further funding was not forthcoming. This work represents the last study (to date) to be completed with the Oxford MSBS.

\section{Current Status and Future Plans}

Following Owen's work in the early-2000s, interest in the MSBS and the LDT as a rarefied flow facility waned. The MSBS was essentially mothballed and with the exception of the work of Wilson et al. [38], ${ }^{\S}$ who adapted LDT to enable development of a wind sensor for the Martian atmosphere, the LDT lay dormant. In 2010 the Oxford Thermofluids

\footnotetext{
${ }^{\S}$ This work was actually conducted in the early-2000s; see Wilson [39].
} 


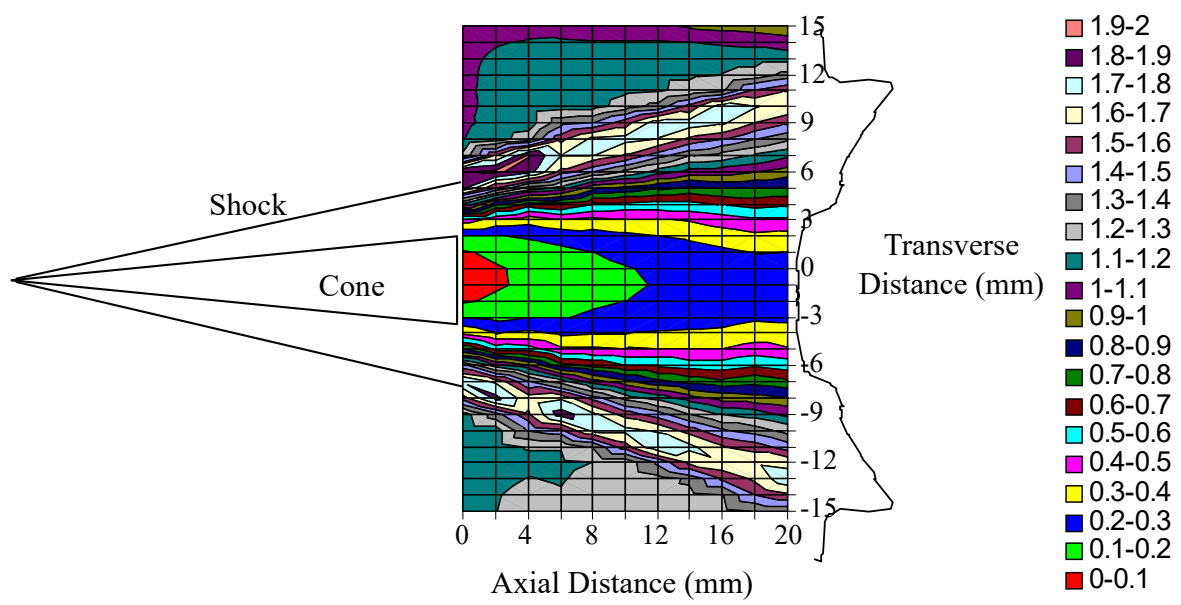

(a) Horizontal symmetry plane contour plot. Model: sharp nose, $6.5^{\circ}$ cone, $5 \mathrm{~mm}$ base diameter. Test condition: $M=5.4, K n_{\infty, d}=0.028$.

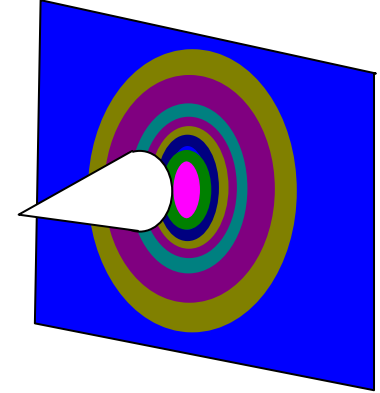

$0^{\circ} \mathrm{AOA}$
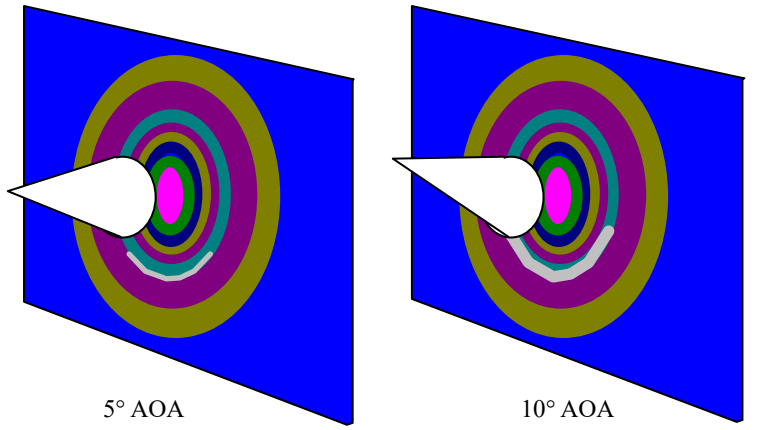

(b) Vertical plane contour plots for different angles of attack. Model: blunt nose, $5^{\circ}$ cone, $5 \mathrm{~mm}$ base diameter. The nose bluntness was $r_{n} / r_{d}=0.375$.

Fig. 19. Normalised pitot pressure survey results in the wake behind a cone. The normalisation is with respect to the freestream pitot pressure. Reproduced from Owen [14].

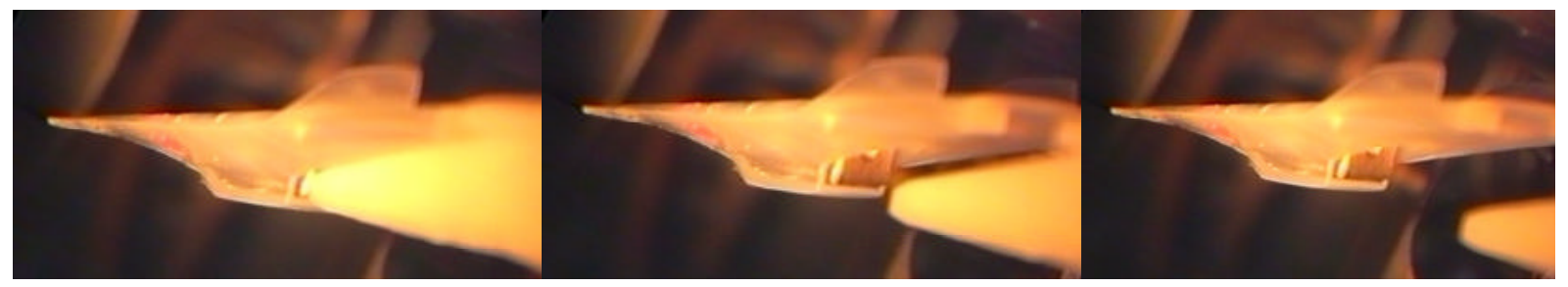

Fig. 20. Wind-off stage separation of the $X-43 A$ vehicle and booster stack using the second generation MSBS. Reproduced from Owen and Owen [35]. 
Institute (aka Osney Lab) shifted into a new premises which required moving several large scale wind tunnel facilities, including the LDT. Refurbishment and recommissioning of the LDT began in 2014 as part of the National Wind Tunnel Facility (NWTF). The NWTF was set up as a UK national effort to invest in wind tunnel technology across a range of speeds and scales. Funding was provided via the Engineering and Physical Sciences Research Counciland the Aerospace Technology Institute.This funding has led to a resurgence of hypersonic wind tunnel testing at Oxford with the development of the T6 Stalker Tunnel [40, 41], a high-enthalpy multi-mode shock tunnel facility, the High Density Tunnel [42], a cold aerodynamic flow facility and, finally, the Low Density Tunnel which completes the trio of NWTF facilities at Oxford.

As reported in Donaldson [16], refurbishment and recommissioning of the LDT as a rarefied flow facility is now complete. Our future plans include increasing the total temperature capability to $>1000 \mathrm{~K}$ and developing a suite of contoured nozzles using modern design methodologies [43]. Noting that the second generation MSBS has a proven capability for accurately measuring milli-Newton magnitude forces, we believe that this balance and MSBSs in general, have a key role to play in improving our understanding the high altitude aerothermodynamics of re-entering satellites and space debris. The MSBS has been removed from storage in preparation for refurbishment and recommissioning over the coming year/s. We plan to upgrade all associated electronics to meet current safety standards and to improve the capability. This includes using new power amplifiers and modern (digital) control and data acquisition systems. Four power amplifiers from AE Techron (1 x PNo. 7724 and 3 x PNo. 7796) have been purchased for this purpose. By undertaking this refurbishment, we aim to eventually expand the use and capability of MSBSs and the LDT beyond what has already been achieved within our group since the early 1970s.

\section{Conclusion}

This paper has presented the history of MSBSs at Oxford over the period late-1960s to 2003. Beginning with the work of Altmann, who developed the first generation balance to suspend spheres, and continuing with Hadjimichalis, who used the balance to complete an extensive study of sphere drag, including the effect of wall-to-total temperature ratio. The second generation balance, a three-component system designed by Haslam-Jones was described as were the subsequent experiments of Dahlen and Smith and Lord who used the new balance to study cone drag in rarefied hypersonic flows. Finally, key improvements made by Owen to the second generation balance which allow non-conical and non-axisymmetric geometries to be suspended were described. Throughout the history of MSBS at Oxford, the primary focus has been the aerodynamic research enabled by MSBSs, rather than the continual advancement of MSBS techniques and technologies as in other groups [44]. The research conducted at Oxford has demonstrated that MSBSs provide the only realistic means to accurately measure milli-Newton magnitude forces on small models in rarefied hypersonic slip, transition and free-molecular flows, and as such have a key role to play in high-altitude, high-speed aerothermodynamic research.

\section{Acknowledgements}

Being an historical review, this paper would not exist but for the original efforts of H. Altmann, M. Hadjimichalis, T. F. Haslam-Jones, G. Dahlen and A. K. Owen. Their work is a source of inspiration and forms a legacy that we hope to reinvigorate and build on. The authors also acknowledge the UK National Wind Tunnel Facility (NWTF) and EPSRC for their support of the Low Density Tunnel under grant EP/L024888/1.

\section{References}

[1] Haslam-Jones, T. F., "Measurements of the Drag of Slender Cones in Hypersonic Flow at Low Reynolds Numbers using a Magnetic Suspension and Balance,” DPhil thesis, University of Oxford, Department of Engineering Science, Oxford, UK, 1977. Also Dept. of Eng. Sci. Report 1235/78 (1978).

[2] Tournier, M., and Laurenceau, P., "Suspension Magnetique d'une Maquette en Soufflerie. (Magnetic Suspension of a Model in a Wind Tunnel.)," La Recherche Aeronautique, Vol. 59, 1957, pp. 21-27.

[3] Crain, C. D., Brown, M. D., and Cortner, A. H., "Design and Initial Calibration of a Magnetic Suspension System for Wind Tunnel Models,” Technical Report AEDC-TR-65-187, Von Karman Gas Dynamics Facility, Arnold Engineering Development Center, 1965.

\footnotetext{
Ihttp://www.nwtf.ac.uk/html/index.html
} 
[4] Altmann, H., "The Measurement of Sphere Drag in Low Density Supersonic Flow by Means of a Magnetic Suspension System," DPhil thesis, University of Oxford, Oxford, UK, 1970.

[5] Tuttle, M. H., Moore, D. L., and Kilgore, R. A., "Magnetic Suspension and Balance System: A Comprehensive, Annotated Bibliography," Technical Memorandum 4318, NASA, Aug. 1991.

[6] Goodyer, M. J., "The Magnetic Suspension of Wind Tunnel Models for Dynamic Testing," PhD thesis, University of Southampton, Department of Aeronautics and Astronautics, Southampton, UK, Dec. 1967.

[7] Crane, R. I., “Studies of Near Wakes in Hypersonic Flow," DPhil thesis, University of Oxford, Department of Engineering Science, Oxford, UK, 1968.

[8] Bird, G. A., "Forty Years of DSMC, and Now?" AIP Conference Proceedings, Vol. 585, No. 1, 2001, pp. 372-380. https://doi.org/10.1063/1.1407585, presented at 22nd International Symposium on Rarefied Gas Dynamics.

[9] Rajasooria, G. P. D., “A Theoretical and Experimental Study of Wakes in Low Density Flow," DPhil thesis, University of Oxford, Department of Engineering Science, Oxford, UK, Sep. 1969.

[10] Altmann, H., "A Magnetic Suspension System for the Measurement of Sphere Drag in Low Density Supersonic Flow," Department of Engineering Science Report 1062/73, University of Oxford, 1973.

[11] Hadjimichalis, M., "A Study of Sphere Drag in the Transition from Continuum to Free Molecular Flow," DPhil thesis, University of Oxford, Department of Engineering Science, Oxford, UK, Oct. 1973. Also Dept. of Eng. Sci. Report 1073/73 (1973).

[12] Dahlen, G., "Cone Drag in the Transition from Continuum to Free Molecular Flow," DPhil thesis, University of Oxford, Department of Engineering Science, Oxford, UK, Mar. 1984. Also Dept. of Eng. Sci. Report 1538/84 (1984).

[13] Smith, R. W., and Lord, R. G., "Drag and Lift Measurements on Inclined Cones Using a Magnetic Suspension and Balance," Rarefied Gas Dynamics: Theoretical and Computational Techniques, Progress in Astronautics and Aeronautics, Vol. 118, edited by D. P. Weaver, D. H. Campbell, and E. P. Muntz, AIAA, 1989, pp. 493-499. https://doi.org/10.2514/5.9781600865923.0493.0499.

[14] Owen, A. K., "Experimental Studies of the Hypersonic, Low Density, Aerodynamics of Re-entry Vehicles," DPhil thesis, University of Oxford, Department of Engineering Science, Oxford, UK, 1997.

[15] Donaldson, N., Doherty, L. J., Ivison, W., Wilson, C. F., McGilvray, M., and Ireland, P. T., "Refurbishment and Characterisation of the Oxford Low Density Hypersonic Wind Tunnel," International Conference on Flight Vehicles Aerothermodynamics and Re-entry Mission \& Engineering (FAR), ESA, Manopoli, Italy, 2019.

[16] Donaldson, N., "Hypersonic Modelling and Testing of Space Debris During Planetary Entry," DPhil thesis, University of Oxford, Department of Engineering Science, Oxford, UK, 2019.

[17] Liu, J. T. C., "The effect of wall temperature on the low Reynolds number hypersonic stagnation region shock layer," International Journal of Heat and Mass Transfer, Vol. 10, No. 11, 1967, pp. 83-95. https://doi.org/10.1016/0017-9310(67)90184-6.

[18] Pavlov, B. M., "Calculation of supersonic flow past blunt bodies using the complete Navier-Stokes equations," Fluid Dynamics, Vol. 3, No. 3, 1968, pp. 88-90. https://doi.org/10.1007/BF01019908.

[19] Wainwright, J. B., "Experimental investigation of the density flow field in the stagnation region of a blunt body in high speed rarefied flow,” Tech. Rep. BD-339-101, Celestial Research Corporation, Nov. 1966.

[20] Lee, D.-K., Lee, J.-S., Han, J.-H., Kawamura, Y., and Chung, S.-J., "Development of a Simulator of a Magnetic Suspension and Balance System," International Journal of Aeronautical \& Space Science, Vol. 11, No. 3, 2010, pp. $175-183$. https://doi.org/10.5139/ijass.2010.11.3.175.

[21] Kaymak, Z. P., "A Simulation of Magnetic Suspension and Balance System for Wind Tunnel," M.sc thesis, The Pennsylvania State University, College of Engineering, Pennsylvania, USA, Dec. 2018. URL https://etda.libraries.psu.edu/catalog/15992zpk5.

[22] Eskins, J., “An Investigation into Force/Moment Calibration Techniques Applicable to a Magnetic Suspension and Balance System," M.Phil thesis, University of Southampton, Department of Aeronautics and Astronautics, Southampton, UK, Aug. 1988. Also NASA CR-181695.

[23] Okuizumi, H., Sawada, H., Nagaike, H., Konishi, Y., and Obayashi, S., "Introduction of 1-m MSBS in Tohoku University, New Device for Aerodynamic Measurement of the Sports Equipment," Proceedings, Vol. 2, No. 6, 2018, p. 273. https: //doi.org/10.3390/proceedings2060273.

[24] Schoenenberger, M., Cox, D. E., Schott, T. D., Mackenzie, A. I., Ramirez, O., Britcher, C. P., Neill, C., Weinmann, M., and Johnson, D., "Preliminary Aerodyanmic Measurements from a Magnetic Suspension and Balance System in a Low-Speed Wind Tunnel," 2018 Applied Aerodynamics Conference, AIAA 2018-3323, Atlanta, Georgia, 2018. https://doi.org/10.2514/6.2018-3323. 
[25] Luh, P. B., Covert, E. E., Whitaker, H. P., and Haldeman, C. W., "Application of Digital Control to a Magnetic Model Suspension and Balance Model,” Contractor Report CR-145316, NASA, Langley Research Center, Jan. 1978.

[26] Ashley, H., "Applications of the Theory of Free Molecule Flow to Aeronautics," Journal of Aeronautical Sciences, Vol. 16, No. 2, 1949, pp. 95-104. https://doi.org/10.2514/8.11735.

[27] Ashkenas, H., and Sherman, F. S., "Structure and Utilization of Supersonic Free Jets in Low Density Wind Tunnels," Contractor Report CF-60423, NASA, Jan. 1965.

[28] Monchick, L., and Mason, E. A., “Transport Properties of Polar Gases,” The Journal of Chemical Physics, Vol. 35, No. 5, 1961, pp. 1676-1697. https://doi.org/10.1063/1.1732130.

[29] Hui, W. H., “Simple Formulae for Supersonic Flow past a Cone,” The Aeronautical Quarterly, Vol. 26, No. 1, 1975 , pp. 11-19. https://doi.org/10.1017/S0001925900007150.

[30] Hirschfelder, J. O., Curtiss, C. F., and Bird, R. B., Molecular Theory of Gases and Liquids, John Wiley \& Sones, In., New York, USA, 1954.

[31] Dahlen, G. A., Macrossan, M. N., Brundin, C. L., and Harvey, J. K., "Blunt cones in rarefied hypersonic flow: Experiments and Monte-Carlo simulations," Proceedings of the 14th International Symposium on Rarefied Gas Dynamics, Vol. I, edited by H. Oguchi, University of Tokyo Press, Tsukuba Science City, Japan, 1984, pp. 229-240.

[32] Garbutt, K. S., and Goodyer, M. J., "The Simulation of a Propulsive Jet and Force Measurement using a Magnetically Suspended Wind Tunnel Model," Second International Symposium on Magnetic Suspension Technology, edited by N. J. Groom and C. P. Britcher, NASA CP-3247, Seattle, Washington, USA, 1994, pp. 291-305.

[33] Owen, A. K., Jones, T. V., and Simmons, R. S., "The Drag of Axially Aligned Cylinders and Cones at Angles of Attack in a Low Density Hypersonic Flow," Proceedings of the 19th International Symposium on Rarefied Gas Dynamics, edited by J. Harvey and L. Gordon, Oxford University Press, Oxford, UK, 1994.

[34] Owen, A. K., “Application of Magnetic Suspension and Balance Systems to Hypersonic Wind Tunnel Testing," 5th International Symposium on Magnetic Suspension Technology, edited by N. J. Groom and C. P. Britcher, NASA CP-2000-210291, Santa Barbara, California, USA, 1999, pp. 171-183.

[35] Owen, A. K., and Owen, F. K., "Magnetic Suspension and Balance Testing in Support of Hyper-X," 12th AIAA International Space Planes and Hypersonic Systems and Technologies Conference, AIAA 2003-6958, Norfolk, Virginia, USA, 2003. https://doi.org/10.2514/6.2003-6958.

[36] Gilmore, M. R., Owen, A. K., and Jones, T. V., "70 Aerobrake Vehicle plus Afterbody in Hypersonic Rarefied Nitrogen Flow," Proceedings of the 19th International Symposium on Rarefied Gas Dynamics, edited by J. Harvey and L. Gordon, Oxford University Press, Oxford, UK, 1994.

[37] Peebles, C., Eleven Seconds into the Unknown: A History of the Hyper-X Program, Library of Flight, American Institute of Aeronautics and Astronautics, Inc., Reston, Virginia, 2011.

[38] Wilson, C. F., Camilletti, A. L., Calcutt, S. B., and Ligrani, P. M., "A wind tunnel for the calibration of Mars wind sensors," Planetary and Space Science, Vol. 56, No. 11, 2008, pp. 1532-1541. https://doi.org/10.1016/j.pss.2008.05.011.

[39] Wilson, C. F., "Measurement of wind on the surface of Mars," DPhil thesis, University of Oxford, Department of Physics, Oxford, UK, 2003.

[40] Collen, P. L., Doherty, L. J., Subiah, S. D., Hyslop, A., and McGilvray, M., "Performance Capability Experiments in T6: A Hypervelocity, Transient and Multi-mode Ground Test Facility," International Conference on Flight Vehicles Aerothermodynamics and Re-entry Mission \& Engineering (FAR), ESA, 2019.

[41] Subiah, S. D., Collen, P. L., Doherty, L. J., , Penty Geraets, R., Hyslop, A., and McGilvray, M., "Condition Development and Commissioning of the Oxford T6 Stalker Tunnel in Reflected Shock Tunnel Mode," International Conference on Flight Vehicles Aerothermodynamics and Re-entry Mission \& Engineering (FAR), ESA, 2019.

[42] Wylie, S., Doherty, L. J., and McGilvray, M., "Commissioning of the Oxford High Density Tunnel for Boundary Layer Stability Measurements at Mach 7," 48th AIAA Fluid Dynamics Conference, AIAA 2018-3074, 2018. https://doi.org/10.2514/6.2018-3074.

[43] Chan, W. Y. K., Jacobs, P. A., Smart, M. K., Grieve, S., Craddock, C. S., and Doherty, L. J., “Aerodynamic Design of Nozzles with Uniform Outflow for Hypervelocity Ground-Test Facilities," Journal of Propulsion and Power, Vol. 34, No. 6, 2018 , pp. 1467 - 1478. https://doi.org/10.2514/1.B36938.

[44] Britcher, C. P., "Application of magnetic suspension technology to large scale facilities - Progress, problems and promises," 35th Aerospace Sciences Meeting and Exhibit, AIAA 97-0346, Reno, NV, USA, 1997. https://doi.org/10.2514/6.1997-346. 